\title{
Nonlinear-in-spin effects in effective-one-body waveform models of spin-aligned, inspiralling, neutron star binaries
}

\author{
Alessandro Nagar, ${ }^{1,2,3}$ Francesco Messina, ${ }^{4,5}$ Piero Rettegno, ${ }^{2,6}$ Donato Bini, ${ }^{7,8}$ \\ Thibault Damour, ${ }^{3}$ Andrea Geralico, ${ }^{7}$ Sarp Akcay, ${ }^{9}$ and Sebastiano Bernuzzi ${ }^{9}$ \\ ${ }^{1}$ Centro Fermi - Museo Storico della Fisica e Centro Studi e Ricerche Enrico Fermi, Rome, Italy \\ ${ }^{2}$ INFN Sezione di Torino, Via P. Giuria 1, 10125 Torino, Italy \\ ${ }^{3}$ Institut des Hautes Etudes Scientifiques, 91440 Bures-sur-Yvette, France \\ ${ }^{4}$ Dipartimento di Fisica, Università degli studi di Milano Bicocca, Piazza della Scienza 3, 20126 Milano, Italy \\ ${ }^{5}$ INFN, Sezione di Milano Bicocca, Piazza della Scienza 3, 20126 Milano, Italy \\ ${ }^{6}$ Dipartimento di Fisica, Università di Torino, via P. Giuria 1, 10125 Torino, Italy \\ ${ }^{7}$ Istituto per le Applicazioni del Calcolo "M. Picone", CNR, I-00185 Rome, Italy \\ ${ }^{8}$ INFN, Sezione di Roma Tre, I-00146 Roma, Italy \\ ${ }^{9}$ Theoretisch-Physikalisches Institut, Friedrich-Schiller-Universität Jena, 07743, Jena, Germany
}

\begin{abstract}
Spinning neutron stars acquire a quadrupole moment due to their own rotation. This quadratic-inspin, self-spin effect depends on the equation of state (EOS) and affects the orbital motion and rate of inspiral of neutron star binaries. Building upon circularized post-Newtonian results, we incorporate the EOS-dependent self-spin (or monopole-quadrupole) terms in the spin-aligned effective-one-body (EOB) waveform model TEOBResumS at next-to-next-to-leading (NNLO) order, together with other (bilinear, cubic and quartic) nonlinear-in-spin effects (at leading order, LO). We point out that the structure of the Hamiltonian of TEOBResums is such that it already incorporates, in the binary black hole case, the recently computed [Levi and Steinhoff, arXiv:1607.04252] quartic-in-spin LO term. Using the gauge-invariant characterization of the phasing provided by the function $Q_{\omega}=\omega^{2} / \dot{\omega}$ of $\omega=2 \pi f$, where $f$ is the gravitational wave frequency, we study the EOS dependence of the self-spin effects and show that: (i) the next-to-leading order (NLO) and NNLO monopole-quadrupole corrections yield increasingly phase-accelerating effects compared to the corresponding LO contribution; (ii) the standard TaylorF2 post-Newtonian (PN) treatment of NLO (3PN) EOS-dependent self-spin effects makes their action stronger than the corresponding EOB description; (iii) the addition to the standard 3PN TaylorF2 post-Newtonian phasing description of self-spin tail effects at LO allows one to reconcile the self-spin part of the TaylorF2 PN phasing with the corresponding TEOBResumS one up to dimensionless frequencies $M \omega \simeq 0.04-0.06$. Such a tail-augmented TaylorF2 approximant then yields an analytically simplified, EOB-faithful, representation of the EOS-dependent self-spin phasing that can be useful to improve current PN-based (or phenomenological) waveform models for inspiralling neutron star binaries. Finally, by generating the inspiral dynamics using the postadiabatic approximation, incorporated in a new implementation of TEOBResums, one finds that the computational time needed to obtain a typical waveform (including all multipoles up to $\ell=8$ ) from $10 \mathrm{~Hz}$ is of the order of $0.4 \mathrm{sec}$.
\end{abstract}

\section{INTRODUCTION}

Neutron stars (NSs) are self-gravitating bodies inside which matter is compressed to very high densities. Gravitational wave (GW) signals can be used to put constraints on the equation of state (EOS) of degenerate matter in these extreme environments. In fact, when a NS is part of a binary system, their mutual tidal interaction deform the stars, affecting the dynamics of the system and the emitted GWs. On August 17, 2017, the first binary neutron star (BNS) inspiral has been detected by the LIGO-Virgo interferometers [1]. One of the important outcomes of this discovery was the measurement of the neutron star radii and EOS from the GW signal $[2,3]$ obtained by extracting from the data the tidal polarizabilities (or deformabilities) related to the NS Love numbers [4-7].

When NSs are spinning, the rate of the inspiral can be modified by an additional EOS-dependent effect, since each NS acquires a quadrupole moment due to its own rotation. The importance of such spin-induced-monopole- quadrupole effects on BNS inspirals was pointed out long ago [8] and recently revived [9] in a data-analysis context, emphasizing that it is important to incorporate such self-spin terms in BNS waveform templates to avoid parameter biases in the case of highly spinning BNS systems. In addition, it was also recently pointed out that self-spin effects might be useful to test the binary black hole nature of the compact objects $[10,11]$. Consistently with these findings, the analysis of GW170817 was done with waveform models that do include EOS-dependent self-spin effects. These were incorporated in resummed form in the SEOBNRv4T [12] and TEOBResums [13] effective one body (EOB) models and in TaylorF2-like post-Newtonian (PN) form in the PhenomPv2NRTidal model $[14,15]$. Both descriptions have their drawbacks and can be improved. On the one hand, the PhenomPv2NRTidal description is incorporating self-spin terms up to next-to-leading order (NLO), but it is biased by the fact that the PN approximation breaks down at some stage in the relativistic regime close to merger. On the other hand, the EOB 
description is robust up to merger, but only the leading order (LO) self-spin effects (both in the EOB Hamiltonian and flux) were included in the models. Although one of the main results of Ref. [15] was to show good consistency between TEOBResums and PhenomPv2NRTidal, this was not a precise apple-with-apple comparison because of the additional NLO self-spin effects included in PhenomPv2NRTidal and not in TEOBResums. Furthermore TEOBResums is actually taking into account an infinite number of self-spin tail terms (in the waveform and flux), that are absent in PhenomPv2NRTidal, so that the precise question about which model is analytically more complete requires an elaborate study. In particular, none of the current waveform models that use a 3.5PN-accurate inspiral description (like TaylorF2 or PhenomPv2NRTidal [15]) are using the EOS-dependent $3.5 \mathrm{PN}$-accurate self-spin tail term, although it is available analytically [13]. Such a term can be obtained by suitably expanding the EOB energy and flux along circular orbits, adapting the procedure of Ref. [16] (see also [7]), that allowed one to cross check the 4.5PN, nonspinning, tail term in the flux formerly obtained from an $a b$ initio PN calculation [17]. Finally, we mention that state-of-theart NR simulations of coalescing BNSs [18-24] are currently barely able to resolve spin-quadratic effects close to merger $[25,26]$ and are too short to measure their cumulative effect during many inspiral orbits. As a consequence, we can only rely on analytical models for their description for LIGO/Virgo targeted analyses.

The purpose of this paper is then to address and answer the questions that remained open in Refs. [13, 15]. We do so by extending the EOS-dependent self-spin sector of TEOBResums to NLO and next-to-next-to-leading order (NNLO), suitably recasting in EOB form recent PN calculations of Levi and Steinhoff [27-29]. For simplicity, this is done in the circular approximation by exploiting the gauge-invariant relation between energy and angular momentum rather than by deriving the explicit canonical transformation that maps the Arnowitt-DeserMisner (ADM) Hamiltonian [30] into the EOB Hamiltonian. This new knowledge allows us to produce a consistent phasing comparison with the TaylorF2 approximant. We find that the phase accelerating effect of the spin-induced quadrupole moment terms is enhanced by the NLO contribution, although the magnitude of the effect as predicted by TEOBResums is always smaller than in the corresponding TaylorF2 description. Remarkably, a TaylorF2 approximant that also incorporates the LO self-spin tail effect yields a self-spin phasing that is essentially equivalent to the NLO TEOBResums one up to frequency $M \omega \simeq 0.05$ independently of the EOS choice. We also show that the LO quartic-in-spin effects entering the circularized Hamiltonian recently computed by Levi and Steinhoff [29] are already contained in the TEOBResumS Hamiltonian of Ref. [31] in the black-hole (BH) case, due to the use of the centrifugal radius. The corresponding correction to the centrifugal radius yielded by the octupolar and hexadecapolar EOS-dependent spin-induced effects (in the non binary BH case) is explicitly obtained.

The paper is organized as follows: Section II builds upon Ref. [13] and describes how spin-quadratic (and spin-quartic) terms are incorporated in the Hamiltonian of TEOBResumS, computing the additional corrections to the centrifugal radius $r_{c}$ [31]. Section III summarizes the spin sector of the, closed form, frequency domain, waveform approximant TaylorF2. The predictions of TEOBResums and of TaylorF2 for what concerns the monopole-quadrupole effects are compared in Sec. IV. Section V collects some concluding remarks. The paper ends with three appendices: Appendix A presents a few suggestions about how incorporating the cubic-in-spin dynamical effects of [29] within the EOB Hamiltonian; Appendix B illustrates the performance of the (highorder) post-adiabatic dynamics, as discussed in Ref. [32], to efficiently compute long-inspiral BNS waveform. In particular, we find that the computational time needed for generating a typical (time-domain) BNS waveform (summed over all multipoles up to $\ell=8$ inclueded) from $10 \mathrm{~Hz}$ is of the order of 0.4 , that goes down to $0.1 \mathrm{sec}$ from $20 \mathrm{~Hz}$. Appendix C re-expresses our results in terms of different spin variables. If not otherwise specified, we use units with $G=c=1$.

\section{NONLINEAR-IN-SPIN EFFECTS WITHIN TEOBRESUMS}

The EOS-dependent self-spin contribution at LO in TEOBResums was discussed extensively in Sec. IIIB of Ref. [13] to which we refer the reader for further details. Our notation follows [13]. We consider binary systems in which the two bodies are labeled by $(A, B)$. Their masses and dimensional spins are denoted $M_{A, B}$ (with $M_{A} \geq M_{B}$ ) and $S_{A, B} \equiv M_{A, B} a_{A, B}$ respectively. The total mass is $M=M_{A}+M_{B}$ and the reduced mass $\mu=\left(M_{A} M_{B}\right) / M$. We also introduce the mass ratio $q \equiv M_{A} / M_{B} \geq 1$, the symmetric mass ratio $\nu=\mu / M$, the mass fractions $X_{A, B} \equiv M_{A, B} / M$ and the shorthand $X_{A B} \equiv X_{A}-X_{B}=\sqrt{1-4 \nu}$. Finally, we make use of the dimensionless spin variables $\tilde{a}_{i} \equiv a_{i} / M \equiv S_{i} /\left(M_{i} M\right)$ together with their symmetric and antisymmetric combinations ${ }^{1} \tilde{a}_{0}=\tilde{a}_{A}+\tilde{a}_{B}$ and $\tilde{a}_{A B}=\tilde{a}_{A}-\tilde{a}_{B}$.

\section{A. Hamiltonian: quadratic-in-spin terms}

In TEOBResums $[13,35]$, which is limited to the case of spin-aligned (nonprecessing) binaries, spin-quadratic effects are treated introducing the "centrifugal radius" $r_{c}$, considered as a function of the Boyer-Lindquist-type

\footnotetext{
1 Note the difference between $\tilde{a}_{i} \equiv a_{i} / M$ and the usually introduced dimensionless spin $\hat{a}_{i} \equiv \chi_{i} \equiv a_{i} / M_{i}$. Note also that in Refs $[13,33,34]$ we had denoted $\tilde{a}_{0}$ as $\hat{a}_{0}$.
} 
EOB radial variable $r$, and of the spin variables. For BBHs, the function $r_{c}\left(r, \tilde{a}_{A}, \tilde{a}_{B}\right)$ incorporates both LO and NLO spin-quadratic effects [35-37]; by contrast only LO spin-quadratic effects were considered for extended objects like NSs [13]. We hence start by generalizing the expression of the centrifugal radius in order to take into account both NLO and NNLO, EOS-dependent, self-spin effects, exploiting the PN-expanded results of Refs. [2729]. The generalized formula for the centrifugal radius that formally takes into account both NLO and NNLO spin-quadratic effects reads

$r_{c}^{2}\left(r, \tilde{a}_{A}, \tilde{a}_{B}\right)^{\mathrm{NNLO}}=r^{2}+\tilde{a}_{Q}^{2}\left(1+\frac{2}{r}\right)+\frac{\delta a_{\mathrm{NLO}}^{2}}{r}+\frac{\delta a_{\mathrm{NNLO}}^{2}}{r^{2}}$,

where we are using a dimensionless radial coordinate $r \equiv$ $\frac{R}{M}$, and we introduced the effective spin variable

$$
\tilde{a}_{Q}^{2} \equiv C_{Q A} \tilde{a}_{A}^{2}+2 \tilde{a}_{A} \tilde{a}_{B}+C_{Q B} \tilde{a}_{B}^{2}
$$

$C_{Q A}$ and $C_{Q B}$ are coefficients that parametrize the quadrupolar deformation acquired by the NSs due to their own rotation. For binary black holes, $C_{Q i}=1$, so $\tilde{a}_{Q}^{2}$ reduces to $\tilde{a}_{0}^{2}$. The parameters $\delta a_{\mathrm{NLO}}^{2}$ and $\delta a_{\mathrm{NNLO}}^{2}$ encode the NLO and NNLO spin-spin information respectively. As mentioned above, working in the circular approximation for simplicity, we compute them exploiting the functional relation between binding energy and orbital angular momentum, that is explicitly given, in PNexpanded form, in Refs. [27-29]. In practice, one computes the PN-expanded EOB dynamics along circular orbits, that will explicitly depend on $\left(\delta a_{\mathrm{NLO}}^{2}, \delta a_{\mathrm{NNLO}}^{2}\right)$, and then fixes these coefficients by comparison with the PNexpanded relation of Ref. [29].

To do so, let us recall the main elements of the Hamiltonian of TEOBResums that are useful for this calculation. Since we are considering nonprecessing systems, the dynamics is described by the dimensionless phasespace variables $\left(r, p_{r_{*}}, \varphi, p_{\varphi}\right)$. We use $\varphi$ to denote the orbital phase, while the (dimensionless) radial and angular momentum are respectively defined as $p_{r_{*}}=P_{R_{*}} / \mu$ and $p_{\varphi}=P_{\varphi} /(\mu M)$. The $\mu$-rescaled EOB Hamiltonian then reads

$$
\hat{H}_{\mathrm{EOB}}=\frac{H_{\mathrm{EOB}}}{\mu}=\frac{1}{\nu} \sqrt{1+2 \nu\left(\hat{H}_{\mathrm{eff}}-1\right)},
$$

where $\hat{H}_{\text {eff }}=\hat{H}_{\text {eff }}^{\text {orb }}+p_{\varphi} \tilde{G}$, i.e., the sum of a orbital (even in spin) and spin-orbit (odd in spin) term. Here $\tilde{G}$ explicitly reads

$$
\tilde{G}=G_{S} \hat{S}+G_{S_{*}} \hat{S}_{*}
$$

where $\hat{S} \equiv\left(S_{A}+S_{B}\right) / M^{2}$ and $\hat{S}_{*} \equiv\left[\left(M_{B} / M_{A}\right) S_{A}+\right.$ $\left.\left(M_{A} / M_{B}\right) S_{B}\right] / M^{2}$. As in previous work, the functions $\left(G_{S}, G_{S_{*}}\right)$ are written in Damour-Jaranowski-Schäfer gauge $[38,39]$, which means gauging away the dependence on the angular momentum $p_{\varphi}$ so that they depend only on $u \equiv 1 / r$ and on $p_{r_{*}}$. The explicit expressions of $\left(G_{S}, G_{S_{*}}\right)$ can be found in Refs. [35, 39]. These expression only retain, in the spin-orbit part of the Hamiltonian, terms that are linear in the spins. However, the complete TEOBResumS model is based on the prescription of Refs. [13, 31, 40] to effectively incorporate, in resummed form, also higher odd-powers of the spins (spin-cubed, $\operatorname{spin}^{5}$ etc.) by suitably replacing the $u$ dependence of the functions $\left(G_{S}, G_{S_{*}}\right)$ with dependence on $u_{c}$. We shall see in Sec. II B below that TEOBResumS delivers a reasonable approximation to the actual LO spin-cubic part of the ADM Hamiltonian of Ref. [29]. In Appendix A we give possible EOB transcriptions of the results of [29].

The orbital part of the effective Hamiltonian reads

$$
\hat{H}_{\mathrm{eff}}^{\mathrm{orb}}=\sqrt{p_{r_{*}}^{2}+A\left(1+p_{\varphi}^{2} u_{c}^{2}+z_{3} p_{r_{*}}^{4} u_{c}^{2}\right)},
$$

with $z_{3}=2 \nu(4-3 \nu)$ and $u_{c} \equiv 1 / r_{c} . \quad A$ is the effective metric potential, whose $\mathrm{PN}$ expansion in the nonspinning limit is

$$
\begin{aligned}
A_{\mathrm{orb}}^{\mathrm{PN}}(u)= & 1-2 u+2 \nu u^{3}+\nu a_{4} u^{4} \\
& +\nu\left(a_{5}^{c}+a_{5}^{\log } \log (u)\right) u^{5}+\mathcal{O}\left[u^{6}\right] .
\end{aligned}
$$

The PN coefficients that appear above explicitly read

$$
\begin{aligned}
a_{4}= & \frac{94}{3}-\frac{41}{32} \pi^{2} \\
a_{5}^{c}= & -\frac{4237}{60}+\frac{2275}{512} \pi^{2}+\frac{256}{5} \log 2+\frac{128}{5} \gamma \\
& -\left(\frac{221}{6}-\frac{41}{32} \pi^{2}\right) \nu, \\
a_{5}^{\log }= & \frac{64}{5}
\end{aligned}
$$

where $\gamma=0.57721 \ldots$ is Euler's constant. In TEOBResums, this effective metric is resummed using a Padé approximant, namely

$$
A_{\text {orb }}(u)=\mathrm{P}_{5}^{1}\left[A_{\text {orb }}^{\mathrm{PN}}(u)\right] .
$$

When spins are present, the metric is built upon the Kerr one and reads

$$
A\left(u ; S_{i}\right)=\frac{1+2 u_{c}}{1+2 u} A_{\mathrm{orb}}\left(u_{c}\right)
$$

We hence start considering circular orbits $\left(p_{r_{*}}=0\right)$ and compute the circular angular momentum, $j$, using the condition $\partial_{u} \hat{H}_{\text {eff }}=0$, that yields the following equation

$$
\begin{aligned}
& \left\{\left[\left(A u_{c}^{2}\right)^{\prime}\right]^{2}-4 A u_{c}^{2}\left(\tilde{G}^{\prime}\right)^{2}\right\} j^{4} \\
& +\left[2 A^{\prime}\left(A u_{c}^{2}\right)^{\prime}-4 A\left(\tilde{G}^{\prime}\right)^{2}\right] j^{2}+\left(A^{\prime}\right)^{2}=0,
\end{aligned}
$$

where the prime indicates $(\cdot)^{\prime} \equiv \partial_{u}(\cdot)$. By expanding the solution of Eq. (10) in series of $u$ and up to the second order in spin one obtains 


$$
\begin{aligned}
j(u)= & \frac{1}{\sqrt{u}}+\frac{3}{2} \sqrt{u}-\frac{3}{8}\left(7 \tilde{a}_{0}+X_{A B} \tilde{a}_{A B}\right) u+\left[\frac{27}{8}-\frac{3}{2} \nu+\tilde{a}_{Q}^{2}\right] u^{3 / 2} \\
& +\left[\left(-\frac{87}{16}+\frac{11}{8} \nu\right) \tilde{a}_{0}-\left(\frac{33}{16}+\frac{1}{8} \nu\right) X_{A B} \tilde{a}_{A B}\right] u^{2} \\
& +\left[\frac{135}{16}+\left(-\frac{433}{12}+\frac{41}{32} \pi^{2}\right) \nu+\frac{441}{128} \tilde{a}_{0}^{2}+\frac{1}{2} \tilde{a}_{Q}^{2}+\left(\frac{9}{128}-\frac{9}{32} \nu\right) \tilde{a}_{A B}^{2}+\frac{63}{64} X_{A B} \tilde{a}_{0} \tilde{a}_{A B}+\frac{5}{4} \delta a_{\mathrm{NLO}}^{2}\right] u^{5 / 2} \\
& +\left[\left(-\frac{63}{4}+\frac{505}{16} \nu+\frac{25}{64} \nu^{2}\right) \tilde{a}_{0}+\left(-\frac{63}{8}+\frac{55}{16} \nu-\frac{5}{64} \nu^{2}\right) X_{A B} \tilde{a}_{A B}\right] u^{3} \\
& +\left[\frac{2835}{128}-\left(\frac{3029}{120}+32 \gamma+\frac{3503}{2048} \pi^{2}+64 \log (2)+16 \log (u)\right) \nu+\left(\frac{539}{12}-\frac{205}{128} \pi^{2}\right) \nu^{2}\right. \\
& +\left(\frac{4095}{256}-\frac{231}{64} \nu\right) \tilde{a}_{0}^{2}-\left(\frac{9}{8}-\frac{9}{4} \nu\right) \tilde{a}_{Q}^{2}+\left(\frac{207}{256}-\frac{51}{16} \nu-\frac{3}{16} \nu^{2}\right) \tilde{a}_{A B}^{2} \\
& \left.+\left(\frac{1017}{128}-\frac{3}{16} \nu\right) X_{A B} \tilde{a}_{0} \tilde{a}_{A B}-\frac{19}{8} \delta a_{\mathrm{NLO}}^{2}+\frac{3}{2} \delta a_{\mathrm{NNLO}}^{2}\right] u^{7 / 2}+\mathcal{O}\left[u^{4}\right] .
\end{aligned}
$$

This truncated series can be inverted so to obtain $u(j)$,

which reads

$$
\begin{aligned}
u(j)= & \frac{1}{j^{2}}+\frac{3}{j^{4}}-\frac{3}{4}\left(7 \tilde{a}_{0}+X_{A B} \tilde{a}_{A B}\right) \frac{1}{j^{5}}+\left(18-3 \nu+2 \tilde{a}_{Q}^{2}\right) \frac{1}{j^{6}} \\
& +\left[\left(-\frac{465}{8}+\frac{11}{4} \nu\right) \tilde{a}_{0}-\left(\frac{87}{8}+\frac{\nu}{4}\right) X_{A B} \tilde{a}_{A B}\right] \frac{1}{j^{7}} \\
& +\left[135+\left(-\frac{311}{3}+\frac{41}{16} \pi^{2}\right) \nu+\frac{441}{8} \tilde{a}_{0}^{2}+22 \tilde{a}_{Q}^{2}+\left(\frac{9}{8}-\frac{9}{2} \nu\right) \tilde{a}_{A B}^{2}+\frac{63}{4} X_{A B} \tilde{a}_{0} \tilde{a}_{A B}+\frac{5}{2} \delta a_{\mathrm{NLO}}^{2}\right] \frac{1}{j^{8}} \\
& +\left[\left(-\frac{1269}{2}+\frac{1273}{8} \nu+\frac{25}{32} \nu^{2}\right) \tilde{a}_{0}+\left(-\frac{531}{4}+\frac{103}{8} \nu-\frac{5}{32} \nu^{2}\right) X_{A B} \tilde{a}_{A B}\right] \frac{1}{j^{9}} \\
& +\left[1134-\left(\frac{163063}{120}+64 \gamma-\frac{31921}{1024} \pi^{2}+128 \log (2)+64 \log (1 / j)\right) \nu+\left(\frac{1321}{12}-\frac{205}{64} \pi^{2}\right) \nu^{2}\right. \\
& +\left(\frac{9009}{8}-\frac{1155}{16} \nu\right) \tilde{a}_{0}^{2}+\left(234-\frac{45}{2} \nu\right) \tilde{a}_{Q}^{2}+\left(\frac{261}{8}-\frac{2073}{16} \nu-\frac{15}{4} \nu^{2}\right) \tilde{a}_{A B}^{2} \\
& \left.+\left(\frac{1557}{4}-\frac{15}{4} \nu\right) X_{A B} \tilde{a}_{0} \tilde{a}_{A B}+29 \delta a_{\mathrm{NLO}}^{2}+3 \delta a_{\mathrm{NNLO}}^{2}\right] \frac{1}{j^{10}}+\mathcal{O}\left[j^{-11}\right] .
\end{aligned}
$$

By placing this expanded expression of $u$ into the EOB Hamiltonian, one can finally obtain the gauge-invariant relation between the binding energy and angular momentum. The binding energy per reduced mass is in fact defined as $E_{b}=(E-M) / \mu$, where $E=\nu \hat{H}_{\mathrm{EOB}}$, and is given as a polynomial in inverse powers of $j$, i.e.,

$$
E_{b}(j)=-\frac{1}{2 j^{2}}\left(1+\sum_{n=1}^{8} \frac{c_{n}}{j^{n}}+\mathcal{O}\left[j^{-9}\right]\right) .
$$

Explicitly, from the expansion of the EOB Hamiltonian along circular orbits we get 


$$
\begin{aligned}
E_{b}(j)= & -\frac{1}{2 j^{2}}\left\{1+\frac{1}{4}(9+\nu) \frac{1}{j^{2}}-\frac{1}{2}\left(7 \tilde{a}_{0}+X_{A B} \tilde{a}_{A B}\right) \frac{1}{j^{3}}\right. \\
& +\frac{1}{8}\left[81-7 \nu+\nu^{2}+8 \tilde{a}_{Q}^{2}\right] \frac{1}{j^{4}}-\frac{3}{8}\left[(81+\nu) \tilde{a}_{0}+(15+\nu) X_{A B} \tilde{a}_{A B}\right] \frac{1}{j^{5}} \\
& +\left[\frac{3861}{64}-\left(\frac{8833}{192}-\frac{41}{32} \pi^{2}\right) \nu-\frac{5}{32} \nu^{2}+\frac{5}{64} \nu^{3}+\frac{441}{16} \tilde{a}_{0}^{2}+\left(\frac{17}{2}+\frac{\nu}{2}\right) \tilde{a}_{Q}^{2}+\left(\frac{9}{16}-\frac{9}{4} \nu\right) \tilde{a}_{A B}^{2}\right. \\
& \left.+\frac{63}{8} X_{A B} \tilde{a}_{0} \tilde{a}_{A B}+\delta a_{\mathrm{NLO}}^{2}\right] \frac{1}{j^{6}}+\frac{1}{16}\left[\left(-4293+822 \nu-5 \nu^{2}\right) \tilde{a}_{0}+\left(-891+42 \nu-5 \nu^{2}\right) X_{A B} \tilde{a}_{A B}\right] \frac{1}{j^{7}} \\
& +\left[\frac{53703}{128}-\left(\frac{989911}{1920}+\frac{128}{5} \gamma-\frac{6581}{512} \pi^{2}+\frac{256}{5} \log (2)+\frac{128}{5} \log (1 / j)\right) \nu+\left(\frac{8875}{384}-\frac{41}{64} \pi^{2}\right) \nu^{2}\right. \\
& -\frac{3}{64} \nu^{3}+\frac{7}{128} \nu^{4}+\left(\frac{14679}{32}-\frac{385}{32} \nu\right) \tilde{a}_{0}^{2}+\left(\frac{603}{8}-\frac{29}{8} \nu+\frac{3}{8} \nu^{2}\right) \tilde{a}_{Q}^{2}+\left(\frac{423}{32}-\frac{1669}{32} \nu-\frac{23}{8} \nu^{2}\right) \tilde{a}_{A B}^{2} \\
& \left.\left.+\left(\frac{2529}{16}+\frac{53}{16} \nu\right) X_{A B} \tilde{a}_{0} \tilde{a}_{A B}+\left(\frac{19}{2}+\frac{\nu}{2}\right) \delta a_{\mathrm{NLO}}^{2}+\delta a_{\mathrm{NNLO}}^{2}\right] \frac{1}{j^{8}}+\mathcal{O}\left[j^{-9}\right]\right\},
\end{aligned}
$$

where we see that the $c_{6}$ and $c_{8}$ coefficients explicitly depend on $\delta a_{\mathrm{NLO}}^{2}$ and $\delta a_{\mathrm{NNLO}}^{2}$. The corresponding quan- tities in Eq. (5.3) of Ref. [28], once expressed in our spin variables $^{2}$, explicitly read

$$
\begin{aligned}
\left(c_{6}^{\mathrm{SS}}\right)^{\mathrm{L}-\mathrm{S}}= & \frac{1}{16}\left\{(375+8 \nu) \tilde{a}_{0}^{2}+8(-23+\nu) \tilde{a}_{Q}^{2}+(7-52 \nu) \tilde{a}_{A B}^{2}+X_{A B}\left[130 \tilde{a}_{0} \tilde{a}_{A B}+16\left(C_{Q A} \tilde{a}_{A}^{2}-C_{Q B} \tilde{a}_{B}^{2}\right)\right]\right\} \\
\left(c_{8}^{\mathrm{SS}}\right)^{\mathrm{L}-\mathrm{S}}= & \frac{1}{112}\left\{-\left(51369-2743 \nu+21 \nu^{2}\right) \tilde{a}_{0}^{2}+\left(13182-1066 \nu+42 \nu^{2}\right) \tilde{a}_{Q}^{2}-\left(5205+6292 \nu+329 \nu^{2}\right) \tilde{a}_{A B}^{2}\right. \\
& \left.+X_{A B}\left[13(1380+7 \nu) \tilde{a}_{0} \tilde{a}_{A B}+(1716+56 \nu)\left(C_{Q A} \tilde{a}_{A}^{2}-C_{Q B} \tilde{a}_{B}^{2}\right)\right]\right\}
\end{aligned}
$$

Comparing Eqs. (15) and (16) to Eq. (14) one obtains

$$
\begin{aligned}
\delta a_{\mathrm{NLO}}^{2}= & -\frac{33}{8} \tilde{a}_{0}^{2}+3 \tilde{a}_{Q}^{2}-\frac{1}{8}(1+4 \nu) \tilde{a}_{A B}^{2} \\
& +X_{A B}\left[\frac{1}{4} \tilde{a}_{0} \tilde{a}_{A B}+\left(C_{Q A} \tilde{a}_{A}^{2}-C_{Q B} \tilde{a}_{B}^{2}\right)\right],
\end{aligned}
$$

\footnotetext{
2 Note that Ref. [28] uses as dimensionless spin variables some quantities, $S_{i}^{\mathrm{L}-\mathrm{S}}$, that correspond to our $S_{i} /\left(M_{A} M_{B}\right)$. Furthermore, their deformation coefficients are denoted by $\left(C_{E S^{2}}, C_{B S^{3}}, C_{E S^{4}}\right)$, in order to highlight the spin order and their electric/magnetic behavior. In our convention, they correspond to $\left(C_{Q}, C_{\mathrm{Oct}}, C_{\mathrm{Hex}}\right)$ respectively, which puts the accent on the multipole of the deformation. We also note that the $\lambda$ constants by Marsat (see Sec. B of Ref. [41]) are the same as Levi and Steinhoff's $C_{B S^{3}}$ and our $C_{\mathrm{Oct}}$ 's.
}

and

$$
\begin{aligned}
\delta a_{\mathrm{NNLO}}^{2}= & -\left(\frac{4419}{224}+\frac{1263}{224} \nu\right) \tilde{a}_{0}^{2}+\left(\frac{387}{28}-\frac{207}{28} \nu\right) \tilde{a}_{Q}^{2} \\
& +\left(\frac{11}{32}-\frac{127}{32} \nu+\frac{3}{8} \nu^{2}\right) \tilde{a}_{A B}^{2} \\
& +X_{A B}\left[-\left(\frac{29}{112}+\frac{21}{8} \nu\right) \tilde{a}_{0} \tilde{a}_{A B}\right. \\
& \left.+\frac{163}{28}\left(C_{Q A} \tilde{a}_{A}^{2}-C_{Q B} \tilde{a}_{B}^{2}\right)\right] .
\end{aligned}
$$

\section{Binary black hole limit}

The $\mathrm{BH}$ case is recovered imposing $C_{Q A}=C_{Q B}=1$ or, equivalently, $\tilde{a}_{Q}^{2}=\tilde{a}_{0}^{2}$ and $\left(C_{Q A} \tilde{a}_{A}^{2}-C_{Q B} \tilde{a}_{B}^{2}\right)=\tilde{a}_{0} \tilde{a}_{A B}$. 
This yields

$$
\begin{aligned}
\delta a_{\mathrm{BBH} \mathrm{NLO}}^{2}= & -\frac{9}{8} \tilde{a}_{0}^{2}-\frac{1}{8}(1+4 \nu) \tilde{a}_{A B}^{2}+\frac{5}{4} X_{A B} \tilde{a}_{0} \tilde{a}_{A B} \\
\delta a_{\mathrm{BBH} \mathrm{NNLO}}^{2}= & -\left(\frac{189}{32}+\frac{417}{32} \nu\right) \tilde{a}_{0}^{2} \\
& +\left(\frac{11}{32}-\frac{127}{32} \nu+\frac{3}{8} \nu^{2}\right) \tilde{a}_{A B}^{2} \\
& +\left(\frac{89}{16}-\frac{21}{8} \nu\right) X_{A B} \tilde{a}_{0} \tilde{a}_{A B} .
\end{aligned}
$$

Eq. (19) agrees with the result for the same quantity obtained in Ref. [35] (see Eq. (60) there) with a different method (see also [42]). The impact of the newly computed $\delta a_{\mathrm{NNLO}}^{2}$ in $\mathrm{BBH}$ systems will be analyzed elsewhere.

\section{B. Hamiltonian: cubic-in-spin terms already included in TEOBResumS}

The LO cubic-in-spin contribution to the PN-expanded Hamiltonian (and thus on the $E_{b}(j)$ curve) was derived in Ref. [28]. This contribution is not fully incorporated in the current version of TEOBResumS. However, one should be aware that some cubic-in-spin terms are already included in the model, because they naturally arise due to the presence of $u_{c}$ in the gyro-gravitomagnetic functions $G_{S}$ and $G_{S_{*}}$ that enter the spin-orbit sector of the Hamiltonian (see Sec. II A). It is then interesting to check how these terms, that are guessed by the resummed structure of the Hamiltonian, do compare with the exact result of Ref. [28]. We now redo the calculations of Sec. II A, this time keeping the cubic-in-spin terms, whose LO enters in the coefficient of $j^{-9}$ in the gauge-invariant relation $E_{b}(j)$. In TEOBResums, the former is given by

$$
\left(c_{7}^{S_{\mathrm{LO}}^{3}}\right)^{\text {TEOBResums }}=-\left(\frac{67}{4} \tilde{a}_{0}+\frac{13}{4} X_{A B} \tilde{a}_{A B}\right) \tilde{a}_{Q}^{2} .
$$

By contrast, the PN-expanded result from Ref. [28] reads

$$
\begin{aligned}
\left(c_{7}^{S_{\mathrm{LO}}^{3}}\right)^{\mathrm{L}-\mathrm{S}}= & -\left(2 C_{\mathrm{Oct} A}+15 C_{Q A}+3 X_{A B} C_{Q A}\right) \tilde{a}_{A}^{3} \\
& -\left[30+21 C_{Q A}+X_{A B}\left(6-3 C_{Q A}\right)\right] \tilde{a}_{A}^{2} \tilde{a}_{B} \\
& -\left[30+21 C_{Q B}-X_{A B}\left(6-3 C_{Q B}\right)\right] \tilde{a}_{A} \tilde{a}_{B}^{2} \\
& -\left(2 C_{\mathrm{Oct} B}+15 C_{Q B}-3 X_{A B} C_{Q B}\right) \tilde{a}_{B}^{3},
\end{aligned}
$$

which is qualitatively different from Eq. (21) above because of the presence of the spin-induced octupolar moments $C_{\text {Oct } A, B}$. Comparing Eqs. (21) and (22), we see that TEOBResumS does not automatically predict (through the definition Eqs. (1), (2) used to incorporate spin-quadratic couplings) the needed PN LO spincubic terms. We have, however, checked that the coeffi- cients entering the two expressions are numerically sufficiently close to lead to nearly equivalent physical predictions. This is especially evident in the BBH case when $C_{\text {Oct } A, B}=C_{Q A, B}=1$. In this case the above equations read

$$
\begin{aligned}
\left(c_{7}^{S_{\mathrm{LO}}^{3}}\right)^{\text {TEOBResumS }} & =-\left(\frac{67}{4} \tilde{a}_{0}+\frac{13}{4} X_{A B} \tilde{a}_{A B}\right) \tilde{a}_{0}^{2}, \\
\left(c_{7}^{S_{\mathrm{LO}}^{3}}\right)^{\mathrm{L}-\mathrm{S}} & =-\left(17 \tilde{a}_{0}+3 X_{A B} \tilde{a}_{A B}\right) \tilde{a}_{0}^{2}
\end{aligned}
$$

with a fractional difference of $1 / 68 \approx 1.47 \%$ between the first coefficients and $1 / 12 \approx 8.3 \%$ for second ones. In practice, the Hamiltonian of TEOBResumS incorporates this approximate description of cubic-in-spin terms, as well as higher-order odd powers of the spins due to its resummed structure. In Appendix A we propose possible EOB transcriptions of the full cubic-in-spin information of Ref. [28].

\section{Hamiltonian: quartic-in-spin terms}

The quartic-in-spin contribution to the PN-expanded $E_{b}(j)$ curve was also computed by Levi and Steinhoff [29]. This corresponds to a $4 \mathrm{PN}$ effect, i.e., it enters at order $1 / j^{10}$. We can thus slightly modify the procedure of Sec. II A above so to apply it also to the recovery of the spin-quartic EOS-dependent terms. We introduce a new parameter $\delta a_{\mathrm{LO}}^{4}$ in the definition of $r_{c}^{2}$ that now reads

$$
r_{c}^{2}=r^{2}+\tilde{a}_{Q}^{2}\left(1+\frac{2}{r}\right)+\frac{\delta a_{\mathrm{NLO}}^{2}}{r}+\frac{\delta a_{\mathrm{NNLO}}^{2}}{r^{2}}+\frac{\delta a_{\mathrm{LO}}^{4}}{r^{2}} .
$$

We then proceed and compute the same formulas we showed before consistently keeping all the quartic-in-spin term. The LO quartic-in-spin term, $O\left(1 / j^{8}\right)$ in $E_{b}(j)$ reads

$$
\left(c_{8}^{S_{\mathrm{LO}}^{4}}\right)^{\mathrm{TEOBResumS}}=3 \tilde{a}_{Q}^{4}+\delta a_{\mathrm{LO}}^{4}
$$

The corresponding term from Ref. [27] reads

$$
\begin{aligned}
\left(c_{8}^{S_{\mathrm{LO}}^{4}}\right)^{\mathrm{L}-\mathrm{S}}= & \frac{3}{4}\left(3 C_{Q A}^{2}+C_{\mathrm{Hex} A}\right) \tilde{a}_{A}^{4} \\
& +3\left(3 C_{Q A}+C_{\mathrm{Oct} A}\right) \tilde{a}_{A}^{3} \tilde{a}_{B} \\
& +9\left(C_{Q A} C_{Q B}+1\right) \tilde{a}_{A}^{2} \tilde{a}_{B}^{2} \\
& +3\left(3 C_{Q B}+C_{\mathrm{Oct} B}\right) \tilde{a}_{A} \tilde{a}_{B}^{3} \\
& +\frac{3}{4}\left(3 C_{Q B}^{2}+C_{\mathrm{Hex} B}\right) \tilde{a}_{B}^{4}
\end{aligned}
$$

where $C_{\text {Oct }}$ and $C_{\text {Hex }}$ are the spin-induced octupolar and hexadecapolar moments quoted above. From these two 
equation one obtains

$$
\begin{aligned}
\delta a_{\mathrm{LO}}^{4}= & \frac{3}{4}\left(C_{\mathrm{Hex} A}-C_{Q A}^{2}\right) \tilde{a}_{A}^{4} \\
& +3\left(C_{\mathrm{Oct} A}-C_{Q A}\right) \tilde{a}_{A}^{3} \tilde{a}_{B} \\
& +3\left(C_{Q A} C_{Q B}-1\right) \tilde{a}_{A}^{2} \tilde{a}_{B}^{2} \\
& +3\left(C_{\mathrm{Oct} B}-C_{Q B}\right) \tilde{a}_{A} \tilde{a}_{B}^{3} \\
& +\frac{3}{4}\left(C_{\mathrm{Hex} B}-C_{Q B}^{2}\right) \tilde{a}_{B}^{4} .
\end{aligned}
$$

To our knowledge $C_{Q}$ and $C_{\text {Oct }}$ have been calculated using numerical approaches [43-45]; by contrast, current knowledge about $C_{\text {Hex }}$ relies on both the slow-rotation approximation (if the NS dimensionelss spin is smaller than 0.3) and on numerical calculations otherwise [46]. All this knowledge (notably recasted in terms of EOS quasi-universal relations [46-48] with the NS Love numbers $[4,6,49-51])$ allows us to evaluate also the impact $\delta a_{\mathrm{LO}}^{4}$ on the BNS phasing. Before doing so, we note that in the $\mathrm{BH}$ limit (when $C_{Q}=C_{\mathrm{Oct}}=C_{\mathrm{Hex}}=1$ ) $\delta a_{\mathrm{LO}}^{4}$ vanishes. It is remarkable that the resummed $\mathrm{EOB}$ Hamiltonian, thanks to the use of the deformed Kerr structure provided by the EOB centrifugal radius [31], is proven to correctly incorporate, at the LO, the quarticin-spin behavior. We also point out, in passing, that the same structure is present also in the EOB Hamiltonian of Refs. [52-54], and thus the quartic-in-spin terms at LO are also present in the SEOBNRv4 corresponding EOB model [55]

\section{Waveform and flux}

Recently, Marsat and Bohé have also computed several terms quadratic in spin entering the post-Newtonian waveform [56]. Their work is yet unpublished, but they kindly gave us access to their most recent results. We report below the corresponding contributions to the factorized waveform amplitude, as the EOS-dependent generalization of Eqs. (39), (43), (44) and (45) of Ref. [34].

$$
\begin{aligned}
\rho_{22}^{\mathrm{SS}, \mathrm{LO}} & =\frac{1}{2} \tilde{a}_{Q}^{2} x^{2} \\
\rho_{22}^{\mathrm{SS}, \mathrm{NLO}} & =\left\{-\frac{187}{252} \tilde{a}_{0}^{2}+\left(\frac{1}{7}+\frac{27}{56} \nu\right) \tilde{a}_{Q}^{2}+\left(\frac{19}{252}-\frac{5}{18} \nu\right) \tilde{a}_{A B}^{2}+X_{A B}\left[\frac{2}{9} \tilde{a}_{0} \tilde{a}_{A B}+\frac{55}{84}\left(C_{Q A} \tilde{a}_{A}^{2}-C_{Q B} \tilde{a}_{B}^{2}\right)\right]\right\} x^{3}, \\
\tilde{f}_{21}^{\mathrm{SS}, \mathrm{LO}} & =\left[-\frac{19}{8} \tilde{a}_{0} \tilde{a}_{A B}-\left(C_{Q A} \tilde{a}_{A}^{2}-C_{Q B} \tilde{a}_{B}^{2}\right)+X_{A B}\left(-\tilde{a}_{0}^{2}+\frac{3}{2} \tilde{a}_{Q}^{2}-\frac{1}{8} \tilde{a}_{A B}^{2}\right)\right] x^{2}, \\
\tilde{f}_{31}^{\mathrm{SS}, \mathrm{LO}} & =\left[-4\left(C_{Q A} \tilde{a}_{A}^{2}-C_{Q B} \tilde{a}_{B}^{2}\right)+\frac{3}{2} X_{A B} \tilde{a}_{Q}^{2}\right] x^{2}, \\
\tilde{f}_{33}^{\mathrm{SS}, \mathrm{LO}} & =\frac{3}{2} X_{A B} \tilde{a}_{Q}^{2} x^{2} .
\end{aligned}
$$

For this work, all these new terms (due to Marsat and Bohé) are incorporated in the flux and waveform of TEOBResumS.
Let us finally comment about the cubic-in-spin terms, that, at leading order, contribute to both the $\ell=m=2$ and to the $\ell=2, m=1$ quadrupolar modes. The corresponding contribution to the flux was obtained by S. Marsat in Ref. [41]. In Ref. [34] this information (though restricted to the $\mathrm{BBH}$ case) was incorporated in the EOB waveform. Although the results of this paper are obtained by omitting such LO spin-cube contribution, let us write here the full terms entering $\rho_{22}^{S}$ and $\tilde{f}_{21}^{S}$, that reduce to part of Eqs. (39) and (43) of Ref. [34] in the black hole limit $C_{Q A}=C_{Q B}=C_{\mathrm{Oct} A}=C_{\mathrm{Oct} B}=1$.

$$
\begin{aligned}
\rho_{22}^{S^{3}}= & \left\{\left(\frac{19}{12} C_{Q A}-C_{\mathrm{Oct} A}-\frac{1}{4} C_{Q A} X_{A B}\right) \tilde{a}_{A}^{3}\right. \\
& +\left[\frac{19}{6}-\frac{17}{12} C_{Q A}-\left(\frac{1}{2}-\frac{1}{4} C_{Q A}\right) X_{A B}\right] \tilde{a}_{A}^{2} \tilde{a}_{B} \\
& +\left[\frac{19}{6}-\frac{17}{12} C_{Q B}+\left(\frac{1}{2}-\frac{1}{4} C_{Q B}\right) X_{A B}\right] \tilde{a}_{A} \tilde{a}_{B}^{2} \\
& \left.+\left(\frac{19}{12} C_{Q B}-C_{\text {Oct } B}+\frac{1}{4} C_{Q B} X_{A B}\right) \tilde{a}_{B}^{3}\right\} x^{7 / 2}, \\
\tilde{f}_{21}^{S^{3}}= & \left(\frac{3}{2} \tilde{a}_{0}^{2}-\frac{3}{4} \tilde{a}_{Q}^{2}\right) \tilde{a}_{A B} x^{5 / 2} .
\end{aligned}
$$




\section{POST-NEWTONIAN PHASING DESCRIPTION}

\section{A. Reminder on the the TaylorF2 phasing approximant}

In the previous section, we have extensively discussed the spin-quadratic (and spin-quartic) contributions in both the Hamiltonian and waveform/flux of TEOBResums. Inspecting the expressions for, e.g., $\left(\delta a_{\mathrm{NLO}}^{2}, \delta a_{\mathrm{NNLO}}^{2}\right)$ one sees that there are several terms that involve $\left(C_{Q A}, C_{Q B}\right)$ and thus take into account the effect due to the spininduced quadrupole moments both in the dynamics and in the radiation (cf. Eqs. (29)-(33)). In this section, we move to the PN-based equivalents of these effects within the TaylorF2 phasing approximant [57]. Although our final goal is to compare the effect of the spin-induced quadrupole moment in TaylorF2 and in TEOBResums, here we aim at being as general as possible. So, for completeness we collect all currently available spin-dependent analytical information that allows us to push the complete spin sector of the TaylorF2 approximant up to 4PN accuracy. This means considering linear and quadratic-inspin effects that also involve tail terms.

Given the energy flux $\mathcal{F}(v)$ and energy $E(v)$ of a binary on circular orbits (expressed as functions of $v \equiv(M \Omega)^{\frac{1}{3}}$, where $\Omega$ is the orbital frequency), the phase of the Fourier transform of the signal is obtained by the following integral (see, e.g., Eq. (3.5) of Ref. [57])

$$
\Psi(f)=2 \pi f t_{\mathrm{ref}}-\phi_{\mathrm{ref}}+2 \int_{v_{f}}^{v_{\mathrm{ref}}}\left(v_{f}^{3}-v^{3}\right) \frac{E^{\prime}(v)}{\mathcal{F}(v)} d v
$$

which assumes the validity of the stationary phase approximation. In short, when this approximation holds, the phase of the time-domain Fourier transform is the Legendre transform of the quadrupolar time domain phase $\phi(t)$, that is given by

$$
\Psi(f)=2 \pi f t_{f}-\phi\left(t_{f}\right)-\pi / 4,
$$

where $t_{f}$ is the solution of the equation $2 \pi f=$ $[d \phi(t) / d t]_{t=t_{f}}$. Using in Eq. (36) the PN-expanded expression for $E^{\prime}(v) / \mathcal{F}(v)$ defines the TaylorF2 phasing approximant. TaylorF2 is typically used at $3.5 \mathrm{PN}$ accuracy for the orbital and spin-orbit part, while the spin-spin part is limited to $3 \mathrm{PN}$ order. It was used in this form for GW data-analysis purposes (see, e.g., [1, 2]). The complete extension of the approximant at $4 \mathrm{PN}$ is currently not possible since the calculation of the $\nu$-dependent part of the energy fluxes is currently incomplete. However, there are higher-order terms in TaylorF2, those involving the tail terms, that are analytically known. For example, Ref. [16] showed how the 4.5PN-accurate term of the energy flux, that is a pure tail term, can be obtained exactly by PN-expanding the EOB energy flux. Applying the same procedure, one can have access to the 3.5PN-accurate, LO, spin-spin tail term as well as to the $4 \mathrm{PN}$-accurate, NLO, spin-orbit tail term. The spin-spin and spin-orbit tail terms in the flux (and TaylorF2) are presented here for the first time. After the integration of Eq. (36) the 4PN-accurate spin-dependent part of the phasing reads

$$
\begin{aligned}
\Psi_{4 \mathrm{PN}, \mathrm{spin}}^{\mathrm{F} 2}(f)= & 2 \pi f t_{c}-\varphi_{c}-\frac{\pi}{4} \\
& +\frac{3}{128 \nu}(\pi f M)^{-5 / 3} \sum_{i=0}^{8} \varphi_{i}(\pi f M)^{i / 3} .
\end{aligned}
$$

As mentioned above, the orbital (spin-independent) part has the same structure, but the $4 \mathrm{PN}$ term is currently incomplete, so we omit its discussion here. Following the procedure of Ref. [16], we construct the PN-expanded total energy flux starting from the EOB-resummed prescription [58]

$$
\mathcal{F}=\sum_{\ell=2}^{\infty} \sum_{\ell=-m}^{m} F_{\ell m}^{\mathrm{Newt}} \hat{F}_{\ell m}
$$

using the orbital dynamical information at the consistent PN order [59-63], the spin information given in Ref. [64] and the new spin waveform results computed by Marsat (Eqs. (18)-(22) from Sec. II D, [16, 34, 65]). The relation between the dynamics, the EOB residual relativistic amplitudes (which can be derived from the PN waveforms) and the flux is given in Ref. [58], and we reproduce it here for completeness:

$$
\hat{F}_{\ell m}=\left(S_{\text {eff }}^{(\epsilon)}\right)^{2}\left|T_{\ell m}\right|^{2}\left(\rho_{\ell m}\right)^{2 \ell}
$$

In this equation, $S_{\mathrm{eff}}^{(\epsilon)}$ is the effective source, that is the effective EOB energy along circular orbits $\hat{E}_{\text {eff }}(x) \equiv E_{\text {eff }} / \mu$ when $\epsilon=0(\ell+m=$ even $)$ or the Newton-normalized orbital angular momentum when $\epsilon=1(\ell+m=$ odd $)$.

\section{B. Extracting tail effects from the EOB resummed tail factor $T_{\ell m}$}

Of crucial importance for our present purpose is the (complex) tail factor $T_{\ell m}$ that resums an infinite number of leading logarithms (see Refs. [58, 66]). This factor automatically incorporates tail effects that can be extracted from it and added to the lower-order PN results.

Expanding the formula (40) multipole by multipole and then summing all the contributions up to $\ell=4$, one obtains the following expression for the 3.5PN spinquadratic tail term in the flux

$$
\hat{\mathcal{F}}_{3.5 \mathrm{PN}}^{\mathrm{SS} \text { tail }}=\left(8 \tilde{a}_{Q}^{2}+\frac{1}{8} \tilde{a}_{A B}^{2}\right) \pi x^{7 / 2},
$$

which reduces to Eq. (26) of Ref. [16] in the BH case, when $\tilde{a}_{Q}^{2}=\tilde{a}_{0}^{2}$. Adding this new piece to Eq. (4.14) of Ref. [64], one obtains a full 3.5PN flux that is used, 
together with the energy given by Eq. (3.33) of the same reference, to compute the 3.5PN accurate spin-spin tail term at NLO (entering the $\varphi_{7}$ coefficient in Eq. (38), as detailed below) by solving the integral given by Eq. (36).

This resummed tail expansion procedure can be applied also to the spin-orbit analogue of the flux. As we did previously, the fact that the EOB-resummed tail amplitude $T_{\ell m}$ contains and infinite amount of $\mathrm{PN}$ information when expanded, using consistently the $\rho_{\ell m}$ and $\tilde{f}_{\ell m}$ information computed from Eqs. (29)-(33) and the pointmass ones from [34, 65], we can compute again Eq. (40), but this time, for what concerns the dynamics, we add to the orbital information of Refs. [59-62] the spin-orbit one of Refs. [41, 67]. The spinning angular momentum at NLO in the spin-orbit coupling is given by Eq. (3.12) of Ref. [68]. This time we use the spin residual relativistic waveform amplitudes up to $\ell=4$, and the purely orbital ones from $\ell=5$ to $\ell=7$, truncating at the right $\mathrm{PN}$ order being careful to account for the relative order of the Newtonian prefactors in the process (see Appendix of [34]). Like in Ref. [16], the $m=$ even flux information of the $\ell=7$ multipoles is out of one PN order with respect to the result we are searching for, so can be neglected in this computation. The new result obtained this way yields ${ }^{3}$

$$
\begin{aligned}
& \mathcal{F}_{5 \mathrm{PN}}^{\mathrm{SO} \text { tail }}=\left[\left(\frac{220103}{1512}+\frac{8421757}{72576} \nu-\frac{9491453}{18144} \nu^{2}\right) \tilde{a}_{0}\right. \\
& \left.+\left(\frac{55499}{3024}+\frac{1149163}{72576} \nu-\frac{4993897}{36288} \nu^{2}\right) X_{A B} \tilde{a}_{A B}\right] \pi x^{5}
\end{aligned}
$$

and gives access to the new $4 \mathrm{PN}$ spin-orbit terms in the $\varphi_{8}$ coefficient in the PN-SPA phase.

\section{Final 4PN-accurate TaylorF2 phasing coefficients}

The complete calculation, at 4PN-accuracy, gives

$$
\begin{aligned}
\varphi_{3}^{\mathrm{SO}}= & \frac{94}{3} \tilde{a}_{0}+\frac{19}{3} X_{A B} \tilde{a}_{A B}, \\
\varphi_{4}^{\mathrm{SS}}= & -50 \tilde{a}_{Q}^{2}-\frac{5}{8} \tilde{a}_{A B}^{2}, \\
\varphi_{5}^{\mathrm{SO}}= & -[1+\log (\pi f M)]\left[\left(\frac{554345}{2268}+\frac{55}{9} \nu\right) \tilde{a}_{0}+\left(\frac{6380}{81}+\frac{85}{9} \nu\right) X_{A B} \tilde{a}_{A B}\right], \\
\varphi_{6}^{\mathrm{SS} ; \mathrm{SO}_{\text {tail }}=} & \pi\left(\frac{1880}{3} \tilde{a}_{0}+130 X_{A B} \tilde{a}_{A B}\right)+\left(\frac{15635}{21}+120 \nu\right) \tilde{a}_{Q}^{2}-\frac{5570}{9} \tilde{a}_{0}^{2} \\
& -\left(\frac{40795}{2016}+\frac{1255}{36} \nu\right) \tilde{a}_{A B}^{2}+X_{A B}\left[-\frac{250}{9} \tilde{a}_{0} \tilde{a}_{A B}+\frac{2215}{12}\left(C_{Q A} \tilde{a}_{A}^{2}-C_{Q B} \tilde{a}_{B}^{2}\right)\right], \\
\varphi_{7}^{\mathrm{SS}} \mathrm{t}_{\text {tail }} ; \mathrm{SO}= & -\pi\left(400 \tilde{a}_{Q}^{2}+\frac{15}{2} \tilde{a}_{A B}^{2}\right)+\left(-\frac{8980424995}{1524096}+\frac{6586595}{1512} \nu-\frac{305}{72} \nu^{2}\right) \tilde{a}_{0} \\
& +\left(-\frac{7189233785}{3048192}+\frac{458555}{6048} \nu-\frac{5345}{144} \nu^{2}\right) X_{A B} \tilde{a}_{A B}, \\
\varphi_{8}^{\mathrm{SO}}= & \pi[1-\log (\pi f M)]\left[\left(\frac{2388425}{2268}-\frac{9925}{27} \nu\right) \tilde{a}_{0}+\left(\frac{1538855}{4536}-\frac{19655}{756} \nu\right) X_{A B} \tilde{a}_{A B}\right],
\end{aligned}
$$

where we have explicitly emphasized in the definition of each term its spin-orbit, spin-spin or spin-tail character. Note that $\varphi_{6}$ and $\varphi_{7}$ receive contributions from both tail and non-tail terms. The $\left(\varphi_{7}^{\mathrm{SS}_{\text {tail }},}, \varphi_{8}^{\mathrm{SO} \text { tail }}\right)$ terms are computed here from the first time.

\footnotetext{
3 By PN consistency, this procedure yields lower PN spin-orbit terms that are well known in literature. Note also that the testparticle limit of (42) (in which $\tilde{a}_{0}=\tilde{a}_{A B} X_{A B}=\tilde{a}_{A}$ ) agrees with the result of Ref. [69], namely $\frac{23605}{144} \tilde{a}_{A} \pi x^{5}$.
}

\section{Isolating the EOS-dependent quadrupole-monopole terms}

From the result above we can finally isolate the EOSdependent quadrupole-monopole terms (i.e., those proportional to $C_{Q i}$ ). These terms are the main focus of the present paper. Multiplying by the Newtonian prefactor (see also [13]) one obtains, at 3.5PN order,

$$
\Psi_{\mathrm{SS}}^{\mathrm{QM}}=\Psi_{\mathrm{SS}}^{\mathrm{QM}, \mathrm{LO}}+\Psi_{\mathrm{SS}}^{\mathrm{QM}, \mathrm{NLO}}+\Psi_{\mathrm{SS}}^{\mathrm{QM}, \text { tail }}
$$


which can be explicitly written as

$$
\begin{aligned}
\Psi_{\mathrm{SS}}^{\mathrm{QM}, \mathrm{LO}}= & -\frac{75}{64 \nu}\left(\tilde{a}_{A}^{2} C_{Q A}+\tilde{a}_{B}^{2} C_{Q B}\right)\left(\frac{M \omega}{2}\right)^{-1 / 3} \\
\Psi_{\mathrm{SS}}^{\mathrm{QM}, \mathrm{NLO}}= & \frac{1}{\nu}\left[\left(\frac{45}{16} \nu+\frac{15635}{896}\right)\left(C_{Q A} \tilde{a}_{A}^{2}+C_{Q B} \tilde{a}_{B}^{2}\right)\right. \\
& \left.+\frac{2215}{512} X_{A B}\left(C_{Q A} \tilde{a}_{A}^{2}-C_{Q B} \tilde{a}_{B}^{2}\right)\right]\left(\frac{M \omega}{2}\right)^{1 / 3} \\
\Psi_{\mathrm{SS}}^{\mathrm{QM}, \text { tail }}= & -\frac{75}{8 \nu} \pi\left(\tilde{a}_{A}^{2} C_{Q A}+\tilde{a}_{B}^{2} C_{Q B}\right)\left(\frac{M \omega}{2}\right)^{2 / 3}
\end{aligned}
$$

where we replaced the circularized quadrupolar gravitational wave frequency $M f$ by $M \omega=2 \pi M f$. The LO $(2 \mathrm{PN})$ term has been derived from the SPA phase originally computed by Poisson [8].

Le us now concentrate on the gauge-invariant description of the phasing of the case of our interest, i.e., Eqs (50), (51) and (52). Following previous practice (see Ref. [13] and references therein) we do so by the $Q_{\omega}$ function that is defined, for any Fourier-domain phase, as

$$
\omega^{2} \frac{d^{2} \Psi(\omega)}{d \omega^{2}}=Q_{\omega}(\omega)
$$

where we identify the time domain and frequency domain circular frequencies, i.e., $\omega_{f}=\omega(t)$. The integral of $Q_{\omega}$ per logarithmic frequency yields the phasing accumulated by the evolution on a given frequency interval $\left(\omega_{L}, \omega_{R}\right)$. The quadrupole-monopole contribution to the PN-expanded $Q_{\omega}$ we are interested in here is given by the following three terms

$$
Q_{\omega}^{\mathrm{SS}_{\mathrm{QM}}}=Q_{\omega}^{\mathrm{SS} \mathrm{QM}, \mathrm{LO}}+Q_{\omega}^{\mathrm{SS}_{\mathrm{QM}, \mathrm{NLO}}}+Q_{\omega}^{\mathrm{SS}_{\mathrm{QM}, \text { tail }}}
$$

that explicitly read

$$
\begin{aligned}
Q_{\omega}^{\mathrm{SS}_{\mathrm{QM}, \mathrm{LO}}}= & -\frac{25}{48 \nu}\left(\tilde{a}_{A}^{2} C_{Q A}+\tilde{a}_{B}^{2} C_{Q B}\right)\left(\frac{\omega}{2}\right)^{-1 / 3}, \\
Q_{\omega}^{\mathrm{SS}}{ }_{\mathrm{QM}, \mathrm{NLO}}= & -\frac{1}{\nu}\left[\left(\frac{5}{8} \nu+\frac{15635}{4032}\right)\left(C_{Q A} \tilde{a}_{A}^{2}+C_{Q B} \tilde{a}_{B}^{2}\right)\right. \\
& \left.+\frac{2215}{2304} X_{A B}\left(C_{Q A} \tilde{a}_{A}^{2}-C_{Q B} \tilde{a}_{B}^{2}\right)\right]\left(\frac{\omega}{2}\right)^{1 / 3},
\end{aligned}
$$$$
Q_{\omega}^{\mathrm{SS}_{\mathrm{QM}, \mathrm{tail}}}=\frac{25}{12 \nu} \pi\left(\tilde{a}_{A}^{2} C_{Q A}+\tilde{a}_{B}^{2} C_{Q B}\right)\left(\frac{\omega}{2}\right)^{2 / 3} .
$$

The aim of the next section will be to investigate how this function compares with the analogous quantity obtained from TEOBResums with all the spin-dependent information detailed in the previous section.

\section{RESULTS: GAUGE-INVARIANT PHASING COMPARISONS OF THE EOS-DEPENDENT SELF-SPIN EFFECTS}

Reference [13] presented a preliminary comparison between the various $\mathrm{PN}$ truncations of the $Q_{\omega}^{\mathrm{SS}_{\mathrm{QM}}}$ written above and the corresponding quantity computed using from the time-domain waveform generated by TEOBResums including only the self-spin information at LO in both the Hamiltonian and waveform/flux, see Fig. 14 there. The main outcome of this preliminary comparison was to show, for an illustrative BNS configuration, the consistency between the PN and EOB descriptions, especially at low frequencies, with the latter being slightly more phase-accelerating than the former. In this respect, Refs. [13, 15] showed the existence of a nonnegligible difference with respect to the TaylorF2 phasing with NLO (i.e., 3PN) self-spin effects. However, Ref. [15], see Sec. VI there, stressed that a more definitive assessment of the EOB/PN performances would need the incorporation of the NLO information in TEOBResums. We shall do so here, closely following what was done in Ref. [13]. To start with, we work at NLO in the self-spin within TEOBResums, adding the corresponding terms to both the Hamiltonian and the multipolar waveform amplitude (and flux). For definiteness, we consider a few BNS configurations, that we list in Table I, ranging from stiffer to softer EOS. Similarly, we mainly explore values of the spins that are compatible with those expected for BNSs. However, to stretch the limits of the model, we also consider a fast-spinning configuration, with $\chi_{A}=0.8$ and $\chi_{B}=0.4$. We note that, although such a configuration is unlikely to exist in a realistic binary system, these spin magnitudes values were considered in the parameter estimation of GW170817 when considering high-spin priors analyses [1, 2].

The $Q_{\omega}(\omega)$ is computed (from the time-domain phasing) in the same way as briefly described in Ref. [13], though here we pushed the lower frequency limit down to $5 \mathrm{~Hz}$, so as to unambiguously identify the frequency region were the $\mathrm{EOB}$ and $\mathrm{PN}$ curve converge together. The code we used to do so is TEOBResums v0.1 that improves over v0.0 (see Ref. [13]) because of the presence of the nonlinear spin terms discussed here ${ }^{4}$ The same terms are also implemented in v1.0 which additionally contains the updated tidal model of Ref. [70] and the post-adiabatic approximation to efficiently compute long inspirals [32] (see Appendix B for additional details). With TEOBResumS v0.1 is is easy to compute such a long waveform with reasonable efficiency. In practice, it is convenient to join together the waveforms computed on three different frequency intervals. The intervals are chosen in a way that the two pieces overlap on a common frequency interval. Table II illustrates our choices

\footnotetext{
4 Note that v0.1 also implements by default the EOS-dependent quartic-in-spin terms of Sec. II C.
} 


\begin{tabular}{lcccccccccccc}
\hline \hline No. & EOS & $M_{A}$ & $M_{B}$ & $q \equiv M_{A} / M_{B}$ & $\chi_{A}$ & $\chi_{B}$ & $\Lambda_{A}$ & $\Lambda_{B}$ & $C_{Q A}$ & $C_{Q B}$ & $M \omega_{\mathrm{mrg}}$ & $\Delta^{\mathrm{EOB}} \phi$ \\
\hline \hline SLy-q1-sA01-sB01 & Sly & 1.35 & 1.35 & 1 & 0.1 & 0.1 & 389.96 & 389.96 & 5.48 & 5.48 & 0.1344 & -2.04 \\
SLy-q1-sA005-sB005 & Sly & 1.35 & 1.35 & 1 & 0.05 & 0.05 & 389.96 & 389.96 & 5.48 & 5.48 & 0.13446 & -0.51 \\
SLy-q1.2-sA005-sA008 & Sly & 1.6573 & 1.354 & 1.224 & 0.05 & 0.08 & 382.7 & 1312.1 & 5.45 & 7.99 & 0.12155 & -1.04 \\
Ms1b-q1-sA01-sB01 & Ms1b & 1.35 & 1.35 & 1 & 0.1 & 0.1 & 1545 & 1545 & 8.40 & 8.40 & 0.10616 & -3.06 \\
Ms1b-q1-sA005-SB005 & Ms1b & 1.35 & 1.35 & 1 & 0.05 & 0.05 & 1545 & 1545 & 8.40 & 8.40 & 0.10616 & -0.76 \\
H4-q1.25-sA005-sB008 & H4 & 1.91 & 1.528 & 1.25 & 0.05 & 0.08 & 499.6 & 1986 & 5.92 & 9.06 & 0.11508 & -1.08 \\
\hline \hline
\end{tabular}

TABLE I. BNS configurations used in this paper. From left to right the columns report: the name of the configurations; the individual masses; the mass ratio; the individual spins, tidal parameters and spin-induced quadrupole moments, that are obtained with the universal relations of $[47,48]$. Then, $M \omega_{\mathrm{mrg}}$ denotes the dimensionless GW frequency at the EOB BNS merger, conventionally defined as the peak of the $\ell=m=2$ waveform amplitude. The last column lists the accumulated phase from $10 \mathrm{~Hz}$ to BNS merger due to the presence of the self-spin effects. For obtaining these numbers only the NLO self-spin terms, both in waveform and Hamiltonian, were included in TEOBResums.
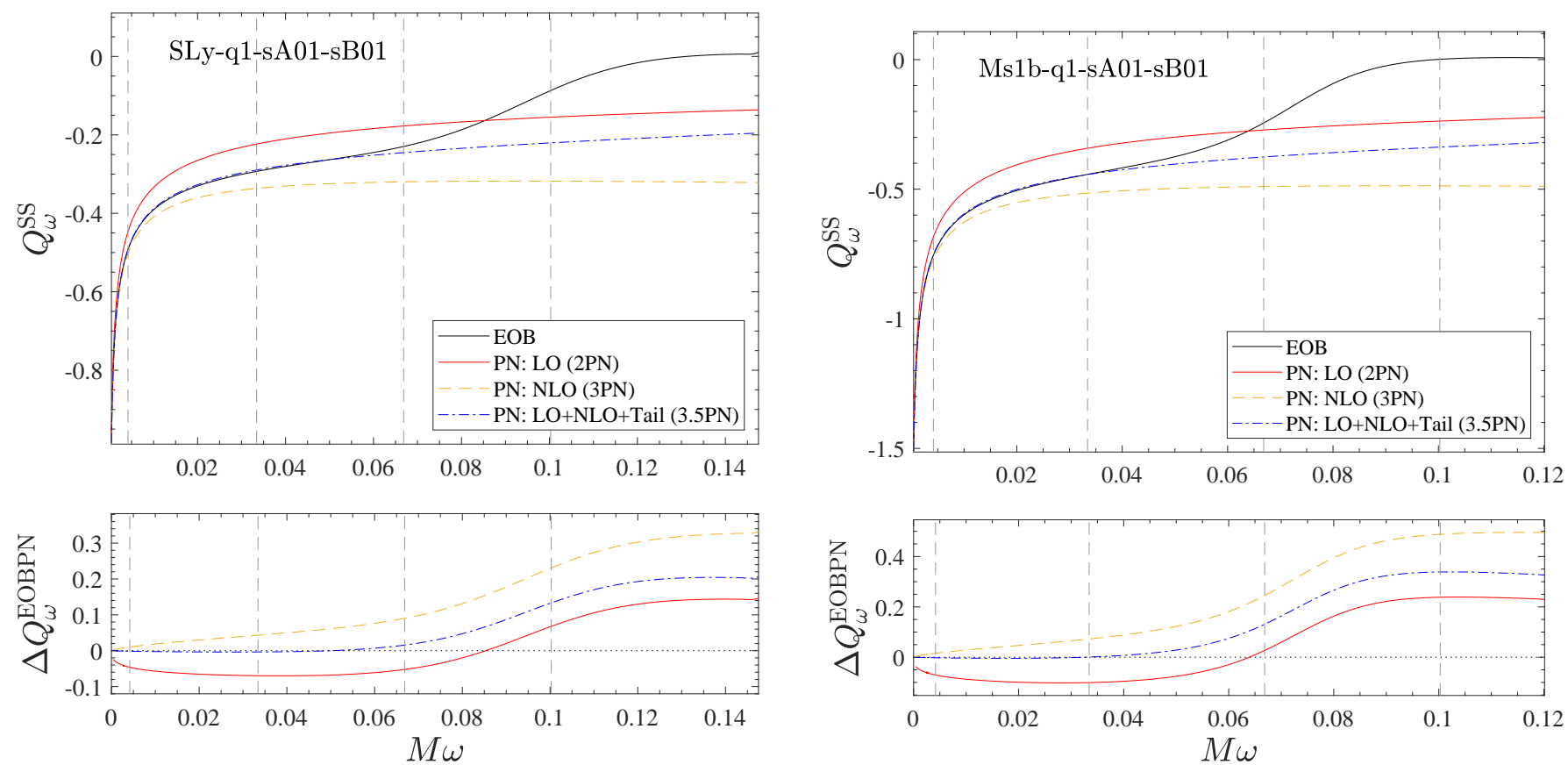

FIG. 1. Left panel: Sly EOS, $M_{A}=M_{B}=1.35, \chi_{A}=\chi_{B}=0.1$ and $C_{Q A}=C_{Q B}=5.48$. Right panel: Ms1B EOS, $M_{A}=M_{B}=1.35, \chi_{A}=\chi_{B}=0.1$ and $C_{Q A}=C_{Q B}=8.40$. The grey vertical lines correspond to 50, 400, 800 and $1200 \mathrm{~Hz}$ respectively. The additional $Q_{\omega}^{\mathrm{SS}_{\mathrm{QM}} \text {,tail }}$ term in TaylorF2 is crucial to get an excellent agreement between the PN-expanded and EOB phasing for most of the inspiral.

for one specific configuration, SLy-q1.2-sA005-sA008 in Table I, and lists: the different running times $\tau$ (including the time needed to write the file on disk); the sampling $\Delta t / M$; and the various intervals (in radius) where the EOB dynamics is evolved. As explained in Ref. [13], in order to explore the low-frequency regime one has to avoid the time-domain oversampling of the waveform that naturally occurs from the ODE solver. To remove this, the raw time-domain waveform phase is additionally downsampled and its derivatives smoothed in order to get a clean and nonoscillatory $Q_{\omega}$ function. The procedure is tedious, but straightforward and it is done separately on different frequency intervals, with the final results eventually joined together. To isolate the, $C_{Q i^{-}}$ dependent only, $Q_{\omega}^{\mathrm{SS}}$ contribution within TEOBResums we perform, for each configuration, two different runs, one with $C_{Q i} \neq 0$ another one with $C_{Q i}=0$. In both cases we compute the time-domain $Q_{\omega}$ and finally calculate

$Q_{\omega}^{\mathrm{TEOBResumS}, \mathrm{SS}}=Q_{\omega}^{\text {TEOBResumS }}{ }_{\mathrm{C}_{\mathrm{Qi}_{\mathrm{i}}} \neq 0}-Q_{\omega}^{\text {TEOBResumS }} \mathrm{C}_{\mathrm{Q}_{\mathrm{i}}=0}$.

Illustrative results are shown in Fig. 1 for the two configurations SLy-q1-sA01-sB01 (left panel) and Ms1b-q1-sA01-sB01 (right panel). Each panel is separated into two subpanels: the top part reports $Q_{\omega}^{\mathrm{SS}}$, with the EOB and the three different PN truncations; 

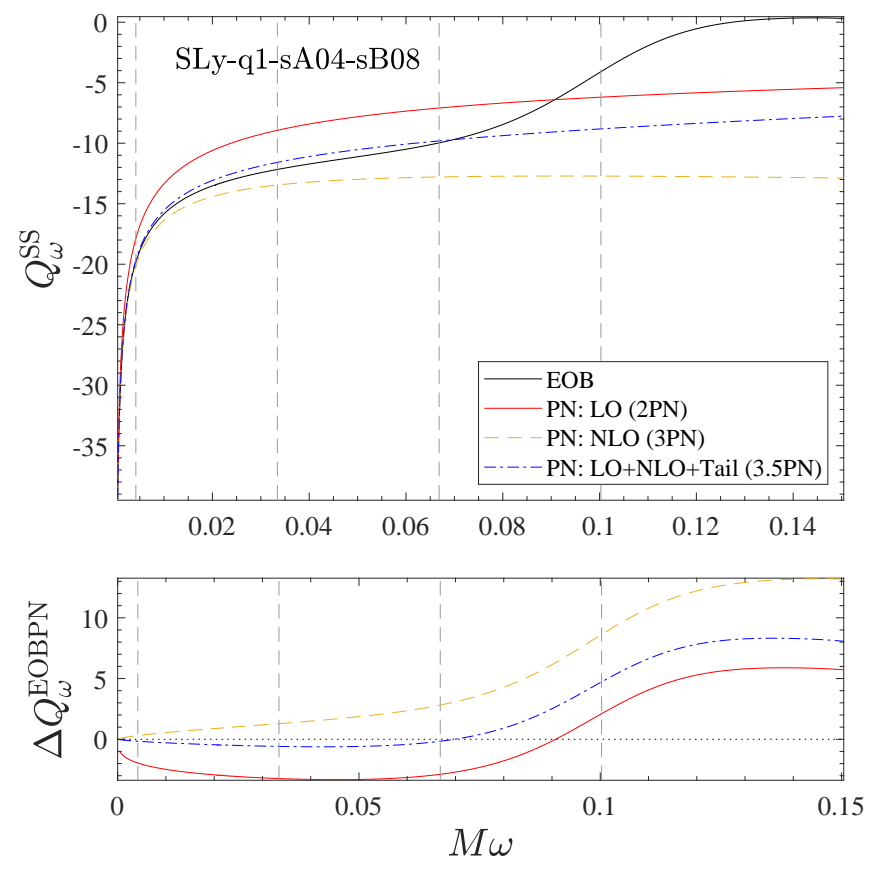

FIG. 2. Closeness of the tail-completed TaylorF2 description of the phasing to the EOB one, even when rather high values of the individual spins are considered.

TABLE II. Configuration SLy-q1.2-sA005-sA008 in Table I. Indicative time, $\tau$, obtained with the v0.1 version of the public implementation of TEOBResums, needed for obtaining the complete waveform, from $5 \mathrm{~Hz}$ up to merger. To ease the computation (and to reduce the high-frequency noise due to the oversampling of the inspiral part), the waveform is computed joining together three pieces (starting at $r_{\max }$ and ending at $r_{\text {min }}$ ) obtained with different sampling rates $\Delta t$. Note that the value of $\tau$ also takes into account the time needed to actually write the data on disk. Runs on an Intel Core i5-8250 $(1.6 \mathrm{GHz})$ and $8 \mathrm{~GB}$ RAM. The code was compiled with the $\mathrm{g}++$ GNU compiler using O3 optimization.

\begin{tabular}{cccccc}
\hline \hline$f_{0}[\mathrm{~Hz}]$ & $r_{\max }$ & $r_{\min }$ & $\Delta t^{-1}[\mathrm{~Hz}]$ & $\Delta t / M$ & $\tau[\mathrm{sec}]$ \\
\hline 5 & 264.11 & 80 & 100 & 674.2 & 102.177 \\
20 & 104.81 & 8 & 10000 & 6.742 & 1.622 \\
200 & 22.58 & merger & 100000 & 0.674 & 1.4832 \\
\hline \hline
\end{tabular}

the bottom panel reports the differences $\Delta Q_{\omega}^{\mathrm{EOBPN}} \equiv$ $Q_{\omega}^{\mathrm{SS}_{\mathrm{EOB}}}-Q_{\omega}^{\mathrm{SS}}$. To orient the reader, the vertical lines superposed to the plot correspond to 50, 400, 800 and $1200 \mathrm{~Hz}$. As mentioned in Ref. [13] the comparison between the time-domain EOB $Q_{\omega}$ and the frequency domain PN-expanded $Q_{\omega}$ is meaningful as long as the SPA holds. In other words, this is true until the adiabatic parameter given by $1 / Q_{\omega}$ is small enough. We will briefly comment about this at the end of the section.

The main conclusions we draw from figure Fig. 1 are: (i) the EOB description of the self-spin effects at NLO

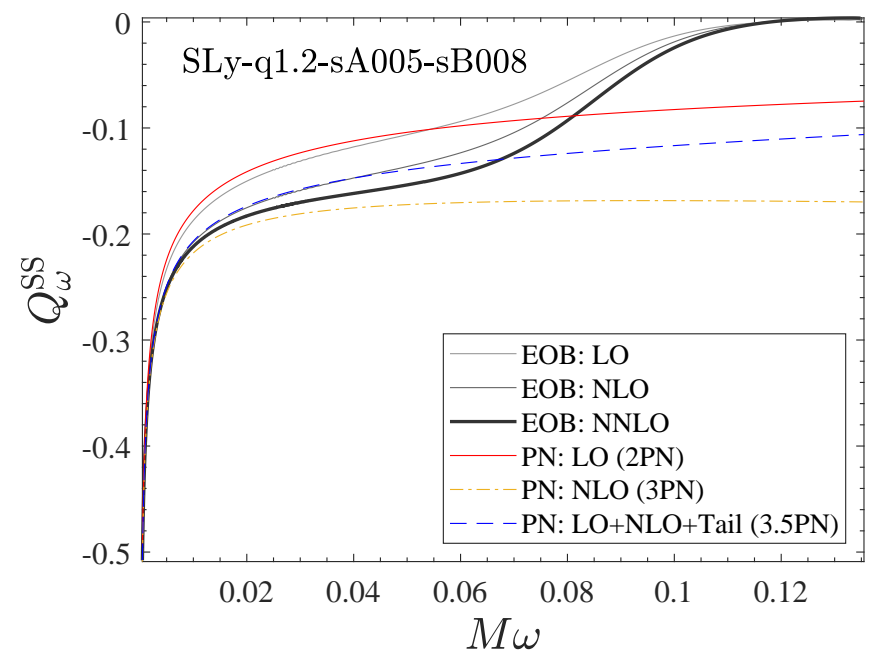

FIG. 3. The effect of the NNLO self-spin term incorporated in the EOB Hamiltonian for one of the configurations of Table I. Although the NNLO term results in an acceleration of the inspiral with respect to the NLO model, the curve is still above the NLO PN-expanded TaylorF2 one.

is more phase-accelerating than the LO one (both PN or EOB, cf. Fig. 14 of Ref. [13]); (ii) it is however less phase-accelerating than the standard TaylorF2 NLO one. This seems to corroborate the suggestion made in Sec. VI of Ref. [15] that part of the phasing accumulated by this approximant is due to its PN-nature. Since, a priori, tidal effects might be degenerate with self-spin effects (since they both accelerate the phasing), the use of the 3PN Taylor-expanded approximant may introduce biases in the measurement of the tidal parameters. This will deserve further investigations in the future; (iii) on the other hand, our comparisons show that the TaylorF2 phasing augmented with the tail factor is fully consistent with the EOB-resummed description up to frequencies $\sim 600 \mathrm{~Hz}$. We have checked that the importance of the self-spin tail term in reconciling the TaylorF2 with the EOB phasing description remains essentially the same when changing the BNS model, though it slightly deteriorates when the individual spins are increased. For example Fig. 2 refers to the SLy-q1-sA04-sB08 configuration, with $\chi_{A}=0.4$ and $\chi_{B}=0.8$, which illustrates the ability of the tail-completed TaylorF2 approximant to reasonably agree with the EOB phasing even in difficult corners of the parameter space.

Let us now explore the implications of the NNLO selfspin correction to $r_{c}$. [We recall that the corrections to $r_{c}$ only enter the Hamiltonian, and do not concern the waveform.] This is done in Fig. 3, which refers to configurations SLy-q1.2-sA005-sB008, i.e., an unequal-mass binary with physically motivated values of the spins and medium values of $\left(C_{Q A}, C_{Q B}\right)$. The NNLO term yields an additional acceleration of the phasing. However, the corresponding modification of the NLO curve is smaller than the modification of the LO curve brought by the NLO contributions. [This intuitively suggests some type 
of convergence of the EOB $Q_{\omega}$ curves as the amount of analytical information is increased.] Since the calculation of the self-spin terms in the energy flux (and waveform) is currently not available at NNLO, we cannot include in Fig. 3 the corresponding TaylorF2 curve. This raises the issue of knowing to which extent the NNLO curve represents a faithful representation of the complete self-spin effects. We can venture an answer based on the knowledge of what happens at NLO. Indeed, in the latter case one finds that the effect of the NLO waveform amplitude terms is almost negligible: the $Q_{\omega}$ curve obtained by switching off these terms is essentially superposed to the one with the NLO waveform corrections. Based on this finding, we expect that a similar situation will hold at the NNLO level.

Let us finally get an idea of the effect of the complete quartic-in-spin term once included into $r_{c}$. This can be done evaluating numerically $C_{\text {Oct }}$ and $C_{\text {Hex }}$ using the quasi-universal fits of Eq. (90) of Ref. [46]. Considering again the configuration SLy-q1.2-sA005-sB008 of Fig. 3 above, one notes that the NNLO spin-square effect is determined by the coefficient $\delta a_{\mathrm{NNLO}}^{2} \simeq 0.108$ entering $r_{c}$. For the same configuration, the quartic-in-spin LO coefficient of Eq. (28), that can be seen as a correction to $\delta a_{\mathrm{NNLO}}^{2}$, numerically reads $\delta a_{\mathrm{LO}}^{4} \simeq 3.05 \times 10^{-4}$, so that its effect would be completely negligible on the phasing analysis of Fig. 3. One also easily checks that one would need to have $\chi_{A}=\chi_{B} \approx 0.29$ so to have $\delta a_{\mathrm{LO}}^{4} \approx 0.1$, thus yielding a phasing correction, at the $Q_{\omega}$ level, comparable to the $\delta a_{\mathrm{NNLO}}^{2}$ displayed in Fig. 3.

\section{CONCLUSIONS}

We have incorporated the EOS-dependent self-spin terms (or monopole-quadrupole effects) in TEOBResums at NLO (i.e., at 3PN order, in both the Hamiltonian and the flux) and at NNLO (4PN order, though only in the Hamiltonian, since the corresponding information in the flux is not available yet). Following previous work [13], this was done through a modification of the centrifugal radius function $r_{c}\left(r, \tilde{a}_{i}\right)$, which now depends on the spininduced quadrupole moments.

Our main findings can be summarized as follows:

(i) Using the $Q_{\omega}$ gauge-invariant description of the phasing, we have found that, once incorporated in the EOB formalism, NLO self-spin effects during the late inspiral are more phase accelerating than the LO ones (consistent with the corresponding PN behavior) but at the same time different and less phase accelerating than the corresponding PN description at NLO expressed through the TaylorF2 approximant. We have verified this to be the case for a few (though illustrative) EOS choices and binary parameters

(ii) The resummed EOB self-spin phasing during the inspiral can be well approximated by augmenting the TaylorF2 approximant by the LO self-spin tail term. The resulting approximant delivers a simple phasing expression that is consistent with the EOB one up to dimensionless frequency up to $M \omega \simeq 0.06$

(iii) In general, the fact that the $\mathrm{PN}$ prediction is always more phase accelerating than the EOB one may have consequences on the estimate of these effects on real data, especially in the case of fast spinning BNS [9] made by recycled NS. This also indicates that current waveform models, notably PhenomPv2_NRTidal [15], that incorporate self-spin effects and that have been used for the analysis of GW170817, should be updated accordingly. This may eventually affect the evaluation of the systematics due to waveform models in the analysis of GW170817.

(iv) Similarly, it will also be interesting to repeat the parameter estimation of GW170817 performed in Ref. [2] using TEOBResumS using the current version of the model that incorporates up to NNLO, EOSdependent, self-spin effects. Note that this will also imply incorporating more, point-mass, spindependent terms than those currently present in the model.

(v) We have illustrated how to consistently compare the EOB and TaylorF2 phasing, notably in the lowfrequency regime, using the $Q_{\omega}(\omega)$ function. In the present paper this comparison was restricted to the quadrupole-monopole part of the phasing. It would be interesting to generalize it to the other parts, so to have in hands precise comparisons between the orbital, spin-orbit or spin-spin parts. We postpone such a comparison to future work.

\section{ACKNOWLEDGMENTS}

F. M. thanks IHES for hospitality during the final stages of development of this work.

\section{Appendix A: Cubic-in-spin terms within the EOB Hamiltonian}

In this Appendix, we discuss preliminary ways of incorporating in the EOB Hamiltonian the LO cubic-in-spin contributions to the dynamics derived in Ref. [28].

As briefly mentioned in the main text, let us first recall that in TEOBResums some contributions cubic in spin are already incorporated in the EOB Hamiltonian via the presence of $u_{c}\left(r, \tilde{a}_{i}\right)$-dependent factors in the gyrogravitomagnetic functions $G_{S}$ and $G_{S_{*}}$ parametrizing the spin-orbit sector of the Hamiltonian (see Sec. II A). Indeed, we have $u_{c}^{2}=u^{2}\left(1+(\right.$ spin - quadratic $\left.) O\left(u^{2}\right)\right)$ so that the linear-in-spin couplings defined by $G_{S}\left(u_{c}\right)$ 
and $G_{S_{*}}\left(u_{c}\right)$ automatically contain some $O\left(u^{5}\right)$ spincubic contributions. However, one checks (see main text, Eqs. (21)-(22)) that the spin-cubic terms thereby already incorporated in the Hamiltonian are not the ones needed from the results of Ref. [28]. This remark suggests a way of incorporating the needed spin-cubic terms in a resummed manner, namely to introduce new definitions of the function $u_{c}\left(r, \tilde{a}_{i}\right)$ to be used as inputs in modified definitions of the gyro-gravitomagnetic functions $G_{S}$ and $G_{S_{*}}$. Say

$$
\begin{aligned}
G_{S} & =2 u u_{c, G_{S}}^{2} \hat{G}_{S}\left(u_{c}\right), \\
G_{S_{*}} & =\frac{3}{2}\left(u_{c, G_{S_{*}}}^{2}\right)^{3 / 2} \hat{G}_{S_{*}}\left(u_{c}\right),
\end{aligned}
$$

where $\hat{G}_{S}\left(u_{c}\right)=1+O\left(u_{c}\right)$ and $\hat{G}_{S_{*}}=1+O\left(u_{c}\right)$ are PN correcting factors [35, 39]. [The arguments $u_{c}$ entering $\hat{G}_{S}\left(u_{c}\right)$ and $\hat{G}_{S_{*}}\left(u_{c}\right)$ can be taken as being any variable such that $u_{c}^{2}=u^{2}\left(1+(\right.$ spin - quadratic $\left.) O\left(u^{2}\right)\right)$.] We found that this possibility a priori involves six parameters, parametrizing the two different spin-quadratic expressions separately entering the modified definitions of $r_{c, G_{S}}^{2}=r^{2}+$ spin - quadratic $(1+O(u))$ and $r_{c, G_{S_{*}}}^{2}=$ $r^{2}+$ spin - quadratic $(1+O(u))$, modelled on Eqs. (1), (2). This leaves the freedom to arbitrarily choose two among these six parameters. This freedom of choice can be used to simplify the resulting definitions. We have explored this avenue. However, at this stage we did not find a unique, convincing way of simplifying the two spinquadratic expressions entering $r_{c, G_{S}}^{2}$ and $r_{c, G_{S_{*}}}^{2}$. We leave further studies along this avenue to future work.

Without committing ourselves to any specific resummed way of incorporating spin-cubic terms in the EOB Hamiltonian, we wish, however, to display here the full information needed for such definitions. To do this we will parametrize the spin-cubic contributions in the following 4-parameter, non-committal form

$$
\begin{aligned}
H_{\mathrm{SO}}= & p_{\varphi}\left[\tilde{G}\left(u, \tilde{a}_{i}\right)+\left(b_{30} \tilde{a}_{A}^{3}+b_{21} \tilde{a}_{A}^{2} \tilde{a}_{B}\right.\right. \\
& \left.\left.+b_{12} \tilde{a}_{A} \tilde{a}_{B}^{2}+b_{03} \tilde{a}_{B}^{3}\right) u^{5}\right],
\end{aligned}
$$

where

$$
\tilde{G}\left(u, \tilde{a}_{i}\right)=2 u^{3} \hat{G}_{S}(u) \hat{S}+\frac{3}{2} u^{3} \hat{G}_{S_{*}}(u) \hat{S}_{*} .
$$

We then follow the procedure described in Section II to determine the four parameters $b_{30}, b_{21}, b_{12}, b_{03}$ entering this parametrization. By calculating the corresponding modified version of $c_{7}^{S_{\mathrm{LO}}^{3}}$ and comparing it to Eq. (21), we obtain the simple expressions

$$
\begin{aligned}
& b_{30}=C_{\mathrm{Oct} A}-3 C_{Q A}, \\
& b_{21}=-6, \\
& b_{12}=-6, \\
& b_{03}=C_{\mathrm{Oct} B}-3 C_{Q B} .
\end{aligned}
$$

Let us note that if we consider the $\mathrm{BH}$ limit where $C_{Q}=C_{\text {Oct }}=1$ the needed modified spin-orbit coupling takes the very simple form

$$
H_{\mathrm{SO} \mathrm{BBH}}=p_{\varphi}\left[\tilde{G}(u)-2\left(\hat{S}+\hat{S}_{*}\right)^{3} u^{5}\right] .
$$

Let us also note that if we insist on utilizing the full TEOBResumS structure, keeping the cubic-in-spin terms that come from the use of $r_{c}\left(r, \tilde{a}_{i}\right)$, as defined in Eq. (1) above, we must modify the expressions of the parameters $b_{30}, b_{21}, b_{12}, b_{03}$ into

$$
\begin{aligned}
& b_{30}^{\prime}=C_{\text {Oct } A}-\frac{7}{8} C_{Q A}-\frac{1}{8} X_{A B} C_{Q A}, \\
& b_{21}^{\prime}=-\frac{7}{4}+\frac{17}{8} C_{Q A}-X_{A B}\left(\frac{1}{4}-\frac{1}{8} C_{Q A}\right), \\
& b_{12}^{\prime}=-\frac{7}{4}+\frac{17}{8} C_{Q B}+X_{A B}\left(\frac{1}{4}-\frac{1}{8} C_{Q B}\right), \\
& b_{03}^{\prime}=C_{\text {Oct } B}-\frac{7}{8} C_{Q B}+\frac{1}{8} X_{A B} C_{Q B} .
\end{aligned}
$$

In that case, the $\mathrm{BBH}$ case leads to the following very simple correction to the spin-sector implied by the current TEOBResumS model:

$$
H_{\mathrm{SO} \mathrm{BBH}}=p_{\varphi}\left[\tilde{G}+\frac{1}{4}\left(\hat{S}+\hat{S}_{*}\right)^{2} \hat{S}_{*} u^{5}\right] .
$$

We postpone a comparison of the various avenues mentioned here to a later work.

\section{Appendix B: Post-adiabatic dynamics}

The EOB/PN comparisons done in the main text employ TEOBResums v0.1, that was implemented in $\mathrm{C}++$. An equivalent, though tidally enhanced model (see [70]) and computationally more efficient version of the model (implemented in C) is v1.0. All our codes are publicly available at

https://bitbucket.org/account/user/eob_ihes/projects/EOB

TEOBResumS v1.0 optionally implements the postadiabatic (PA) approximation to efficiently deal with the long inspiral phase [32]. Following the logic of Appendix $\mathrm{B}$ of Ref. [70], we here present the performance of the PA evolution in the case of spinning neutron stars. The result presented here are obtained incorporating (i) NLO spinquadratic information in the waveform and (ii) NNLO spin-quadratic information in the Hamiltonian. This should be considered as the default choice in TEOBResumS for what concerns spinning BNS. Optionally, it is possible to switch on the EOS-dependence in the quartic-in-spin correction to $r_{c}$, Eq. (28), though this does not come as default choice in the code.

Within TEOBResumS, the dynamics of a binary system is usually determined by numerically solving the four Ordinary Differential Equations (ODEs) of the Hamiltonian 
relative dynamics. The time needed to solve these ODEs usually weighs as the main contribution to the waveform evaluation time. Using this C-implementation of TEOBResumS, a typical time-domain BNS waveform requires $\sim 1$ sec to be generated starting from a GW frequency of $10 \mathrm{~Hz}$ by means of standard Runge-Kutta integration routines with adaptive step-size that are publicly available through the GNU Scientific Library (GSL). Thus, ODE integration as is cannot be used in parameter estimation runs that require the generation of $10^{7}$ waveforms. Reference [32] proposed a method of reducing the evaluation time by making use of the PA approximation to compute the system dynamics. In Ref. [70] we restricted to non spinning BNS and we showed, for the first time, how a waveform obtained from the complete ODE evolution compares with a waveform obtained by stitching the PA dynamics to the complete dynamics for the last few orbits up to merger (as suggested in [32]). For completeness, we here present the same comparison for two illustrative, spinning, BNS systems.

Let us briefly summarize the approach of Ref. [32]. The PA approximation to the EOB dynamics was introduced in Refs. [71, 72] (and expanded in Refs. [73, 74]) and is currently used to initialize the relative dynamics in TEOBResums with negligible eccentricity. Using this approximation, it is possible to analytically compute the radial and angular momentum of a binary system, under the assumption that the radiation reaction force is small. This is true in the early inspiral phase and progressively loses validity when the two objects get close. The approach starts by considering the conservative system, when the flux is null, and then computes the successive corrections to the momenta. We denote as $n \mathrm{PA}$ the $n$-th order iteration of this procedure. Practically,

\begin{tabular}{ccccccc}
\hline \hline$f_{0}[\mathrm{~Hz}]$ & $r_{0}$ & $r_{\min }$ & $N_{r}$ & $\Delta r$ & $\tau_{8 \mathrm{PA}}[\mathrm{sec}]$ & $\tau_{\mathrm{ODE}}[\mathrm{sec}]$ \\
\hline 20 & 112.81 & 12 & 500 & 0.20 & 0.03 & 0.53 \\
10 & 179.02 & 12 & 830 & 0.20 & 0.05 & 1.1 \\
\hline \hline
\end{tabular}

TABLE III. Performance of TEOBResumS v1.0 for a BNS system with $1.35 M_{\odot}+1.35 M_{\odot}$, SLy EOS and $\chi_{A}=\chi_{B}=0.1$. The waveform for the $\chi_{A}=\chi_{B}=-0.1$ case is a little shorter but the evaluation times are comparable to the ones showed in the table. $f_{0}$ and $r_{0}$ denote the initial GW frequency and radial separation. The $8 \mathrm{PA}$ dynamics is computed on a grid with $N_{r}$ points and grid separation $\Delta r$ that ends at $r_{\min }$ and then is completed by the standard ODE one. The evaluation times $\tau$ are determined using a standard Intel Core i7, $1.8 \mathrm{GHz}$ and $16 \mathrm{~GB}$ RAM. The code is compiled with the GNU gcc compiler using O3 optimization.

to compute the PA dynamics, we first build a uniform radial grid from the initial radius $r_{0}$ to an $r_{\text {min }}$ up until which we are sure the approximation holds. We then analytically compute the momenta that correspond to each radius at a chosen PA order. Finally, we determine the full dynamics recovering the time and orbital phase by quadratures. From $r_{\text {min }}$ we can then start the usual ODE-based dynamics using the PA quantities as initial
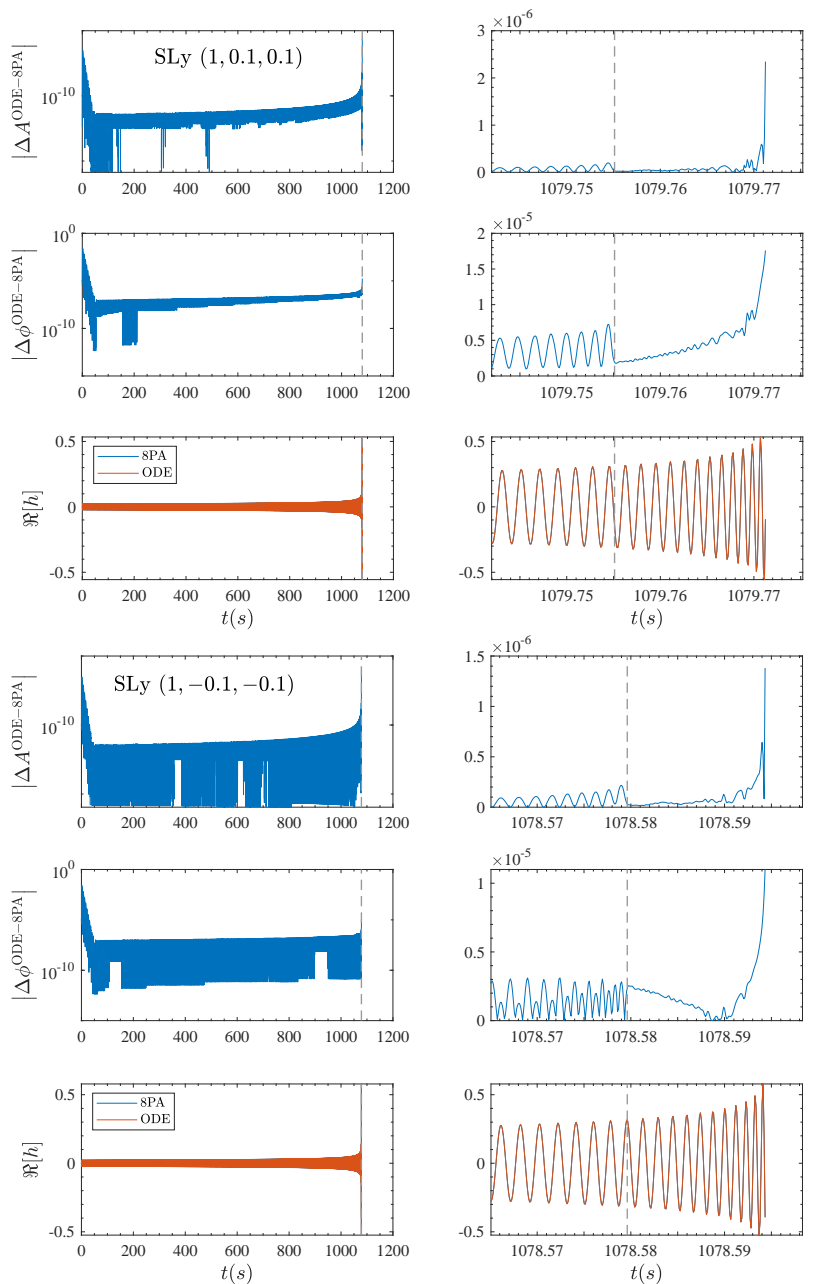

FIG. 4. Comparison between the waveforms (obtained summing all modes up to $\ell_{\max }=8$, see Eq. (B1)) computed solving the ODEs with the GSL rk8 routine with adaptive stepsize and the PA waveform completed with the same ODE solver after $r<r_{\min }$. We have considered BNS systems with $1.35 M_{\odot}+1.35 M_{\odot}$ and SLy EOS (see first row of Table I), starting at initial frequency $10 \mathrm{~Hz}$. Dimensionless spins are $\chi_{A}=\chi_{B}=0.1$ (top) and $\chi_{A}=\chi_{B}=-0.1$ (bottom). The parameters used for the PA run are listed in Table III. The dashed grey line marks the stitching point, $r_{\min }$, between the PA and ODE-based dynamics. Given the waveform strain as $h \equiv A e^{-\mathrm{i} \phi}$, we defined the phase difference as $\Delta \phi^{\mathrm{ODE}-8 \mathrm{PA}} \equiv \phi^{\mathrm{ODE}}-\phi^{8 \mathrm{PA}}$ and the fractional amplitude difference as $\Delta A^{\mathrm{ODE}-8 \mathrm{PA}} \equiv\left(A^{\mathrm{ODE}}-A^{8 \mathrm{PA}}\right) / A^{\mathrm{ODE}}$. The larger differences at the beginning of the evolution are partly due to the fact that the complete ODE is started using only 2PAaccurate initial data.

data as it is usually done (at 2PA order) in TEOBResums. With this method one can avoid to numerically solving two Hamilton equations (those for the momenta), while the orbital phase and time can be obtained by quadratures over a rather sparse radial grid.

Figure 4 displays the performance of the PA approximation (at 8PA order) for two, illustrative, spinning 


\begin{tabular}{cccc}
\hline \hline$f_{0}[\mathrm{~Hz}]$ & $r_{0}$ & $\tau_{8 \mathrm{PA}}^{\text {int }}[\mathrm{sec}]$ & $\tau_{\mathrm{ODE}}^{\text {int }}[\mathrm{sec}]$ \\
\hline 20 & 112.81 & 0.10 & 0.64 \\
10 & 179.02 & 0.37 & 1.70 \\
\hline \hline
\end{tabular}

TABLE IV. Performance of the TEOBResums v1.0 when the final waveform is interpolated on a time grid evenly sampled at $1 /(4096 \mathrm{~Hz})$. We use the standard spline interpolation routine implemented in the GSL library. The considered system coincides with the one of Table III.

BNS systems with $1.35 M_{\odot}+1.35 M_{\odot}$ and SLy EOS. The figure shows the distance-normalized waveform strain $h \equiv \mathcal{R}\left(h_{+}-\mathrm{i} h_{\times}\right)$, where we recall that the multipolar decomposition of the waveform reads

$$
\mathcal{R}\left(h_{+}-\mathrm{i} h_{\times}\right)=\sum_{\ell=2}^{\ell_{\text {max }}} \sum_{m=-\ell}^{\ell} h_{\ell m-2} Y(\theta, \Phi),
$$

where $h_{\ell m}$ are the waveform (complex) multipoles and ${ }_{-2} Y(\theta, \Phi)$ are the $s=-2$ spin-weighted spherical harmonics (that are here evaluated at $\theta=\Phi=0$ ). In fig. 4 we evaluate $h$ with $\ell_{\max }=8$, i.e. retaining in the waveform the same 35 multipoles that are used to compute the EOB radiation reaction. For each binary, each subpanel displays the the waveform fractional-amplitude difference (top), phase difference (medium) and real part of the waveform strain. The left columns offer a global view, while the right columns focus on the last few GW cycles up to merger. The vertical dashed line marks the time where the PA evolution is stitched to the ODE evolution for the last orbits where the PA approximation breaks down. Table III highlights the performances of TEOBResumS v1.0 for such a case. Note that the initial radius is determined by solving the circular Hamilton's equations instead of relying on the Newtonian Kepler's law, as discussed in Sec. VI of Ref. [13].

The waveform computed using the PA dynamics (completed with the ODE for the last few orbits) only takes around 50 milliseconds to be evaluated. Such a time is comparable to the one needed by the surrogate models that are currently being constructed in order to reduce waveform evaluation times (see e.g. [75]) and that, typically, only involve the $\ell=m=2$ mode. Finally, Table IV illustrates the performance of TEOBResumS when the waveform of above, which is obtained on a nonuniform temporal grid (because the corresponding radial grid is evenly spaced), is interpolated on an evenly spaced time grid, sampled at $\Delta t^{-1}=4096 \mathrm{~Hz}$, that is usually neecessary to compute the Fourier transform with standard algorithms. It is remarkable that the generation time of the full multipolar waveform is below $1 \mathrm{sec}$ also when the starting frequency is $10 \mathrm{~Hz}$. Such interpolation is done with the spline interpolant that is freely available in the GSL library and looks to be the main routine responsible for the computational cost of the waveform generation. We expect this can be further speed up exploting vectorization or shared memory parallelization. Similarly, one expects that the number of radial gridpoints needed might be lowered further by adopting a quadrature formula at higher order (now a third-order one is implemented, following Ref. [32]) to recover the orbital phase and time. Such technical improvements will be explored extensively in forthcoming works.

\section{Appendix C: Using $\hat{S}$ and $\hat{S}_{*}$ as spin variables.}

For completeness, we report here the results of Sec. II using $\hat{S}$ and $\hat{S}_{*}$ as spin variables. First, the newly computed $\delta a_{\mathrm{NLO}}^{2}$ and $\delta a_{\mathrm{NNLO}}^{2}$ are written as quadratic forms in $\left(S, S_{*}\right)$ as 


$$
\begin{aligned}
\delta a_{\mathrm{NLO}}^{2}= & \frac{1}{1-4 \nu}\left\{\left[-4+9 \nu+(2-5 \nu)\left(C_{Q A}+C_{Q B}\right)+(2-\nu) X_{A B}\left(C_{Q A}-C_{Q B}\right)\right] \hat{S}^{2}\right. \\
& +\left[-\frac{9}{2}+11 \nu+(1-\nu)\left(C_{Q A}+C_{Q B}\right)-(1+\nu) X_{A B}\left(C_{Q A}-C_{Q B}\right)\right] \hat{S}_{*}^{2} \\
& \left.+\left[-2+22 \nu-6 \nu\left(C_{Q A}+C_{Q B}\right)-2 \nu X_{A B}\left(C_{Q A}-C_{Q B}\right)\right] \hat{S} \hat{S}_{*}\right\} \\
\delta a_{\mathrm{NNLO}}^{2}= & \frac{1}{1-4 \nu}\left\{\left[-\frac{275}{14}+\frac{561}{14} \nu+\frac{675}{14} \nu^{2}\right.\right. \\
& \left.+\left(\frac{275}{28}-\frac{1633}{56} \nu+\frac{207}{28} \nu^{2}\right)\left(C_{Q A}+C_{Q B}\right)+\left(\frac{275}{28}-\frac{533}{56} \nu\right) X_{A B}\left(C_{Q A}-C_{Q B}\right)\right] \hat{S}^{2} \\
& +\left[-\frac{153}{8}+\frac{173}{4} \nu+\frac{381}{14} \nu^{2}+\left(4-\frac{47}{8} \nu+\frac{207}{28} \nu^{2}\right)\left(C_{Q A}+C_{Q B}\right)-\left(4+\frac{17}{8} \nu\right) X_{A B}\left(C_{Q A}-C_{Q B}\right)\right] \hat{S}_{*}^{2} \\
& \left.+\left[-\frac{25}{2}+\frac{4727}{56} \nu-\frac{163}{14} \nu^{2}-\left(\frac{387}{14}-\frac{207}{14} \nu\right) \nu\left(C_{Q A}+C_{Q B}\right)-\frac{163}{14} \nu X_{A B}\left(C_{Q A}-C_{Q B}\right)\right] \hat{S} \hat{S}_{*}\right\}
\end{aligned}
$$

Note that the use of $\left(S, S_{*}\right)$ leads to formally singular terms when $\nu=1 / 4$. This singularity is actually reab- sorbed by $\left(\hat{S}, \hat{S}_{*}\right)$ when the limit is done carefully taking into account the various mass terms. From these equations, one can obtain the orbital angular momentum $j$ as a function of $u$, namely 


$$
\begin{aligned}
& j(u)=\frac{1}{\sqrt{u}}+\frac{3}{2} \sqrt{u}-3\left(\hat{S}+\frac{3}{4} \hat{S}_{*}\right) u \\
& +\left\{-\frac{27}{8}-\frac{3}{2} \nu+\frac{1}{1-4 \nu}\left[-2 \nu+\left(\frac{1}{2}-\nu\right)\left(C_{Q A}+C_{Q B}\right)+\frac{1}{2} X_{A B}\left(C_{Q A}-C_{Q B}\right)\right] \hat{S}^{2}\right. \\
& +\frac{1}{1-4 \nu}\left[-2 \nu+\left(\frac{1}{2}-\nu\right)\left(C_{Q A}+C_{Q B}\right)-\frac{1}{2} X_{A B}\left(C_{Q A}-C_{Q B}\right)\right] \hat{S}_{*}^{2}+ \\
& \left.+\frac{2}{1-4 \nu}\left[1-2 \nu-\nu\left(C_{Q A}+C_{Q B}\right)\right] \hat{S} \hat{S}_{*}\right\} u^{3 / 2}+\left[\left(-\frac{15}{2}+\frac{5}{4} \nu\right) \hat{S}+\left(-\frac{27}{8}+\frac{3}{2} \nu\right) \hat{S}_{*}\right] u^{2} \\
& +\left\{\frac{135}{16}+\left(-\frac{433}{12}+\frac{41}{32} \pi^{2}\right) \nu\right. \\
& +\frac{1}{1-4 \nu}\left[-\frac{1}{2}-\frac{31}{4} \nu+\left(\frac{11}{4}-\frac{27}{4} \nu\right)\left(C_{Q A}+C_{Q B}\right)+\left(\frac{11}{4}-\frac{5}{4} \nu\right) X_{A B}\left(C_{Q A}-C_{Q B}\right)\right] \hat{S}^{2} \\
& +\frac{1}{1-4 \nu}\left[-\frac{99}{32}+\frac{21}{8} \nu+\left(\frac{3}{2}-\frac{7}{4} \nu\right)\left(C_{Q A}+C_{Q B}\right)-\left(\frac{3}{2}+\frac{5}{4} \nu\right) X_{A B}\left(C_{Q A}-C_{Q B}\right)\right] \hat{S}_{*}^{2} \\
& \left.+\frac{1}{1-4 \nu}\left[\frac{21}{4}-\frac{3}{2} \nu-\frac{17}{2} \nu\left(C_{Q A}+C_{Q B}\right)-\frac{5}{2} \nu\left(C_{Q A}-C_{Q B}\right)\right] \hat{S} \hat{S}_{*}\right\} u^{5 / 2} \\
& +\left[\left(-\frac{189}{8}+35 \nu+\frac{5}{16} \nu^{2}\right) \hat{S}+\left(-\frac{63}{8}+\frac{225}{8} \nu+\frac{15}{32} \nu^{2}\right) \hat{S}_{*}\right] u^{3} \\
& +\left\{\frac{2835}{128}-\left(\frac{3029}{120}+32 \gamma+\frac{3503}{2048} \pi^{2}+64 \log (2)+16 \log (u)\right) \nu+\left(\frac{539}{12}-\frac{205}{128} \pi^{2}\right) \nu^{2}\right. \\
& +\frac{1}{1-4 \nu}\left[\frac{67}{14}-\frac{3459}{56} \nu+\frac{2319}{28} \nu^{2}+\left(\frac{1055}{112}-\frac{3317}{112} \nu+\frac{495}{56} \nu^{2}\right)\left(C_{Q A}+C_{Q B}\right)\right. \\
& \left.+\left(\frac{1055}{112}-\frac{1207}{112} \nu\right) X_{A B}\left(C_{Q A}-C_{Q B}\right)\right] \hat{S}^{2}+\frac{1}{1-4 \nu}\left[-\frac{585}{64}+\frac{35}{16} \nu+\frac{1395}{28} \nu^{2}\right. \\
& \left.+\left(\frac{49}{16}-\frac{67}{16} \nu+\frac{495}{56} \nu^{2}\right)\left(C_{Q A}+C_{Q B}\right)-\left(\frac{49}{16}+\frac{31}{16} \nu\right) X_{A B}\left(C_{Q A}-C_{Q B}\right)\right] \hat{S}_{*}^{2} \\
& +\frac{1}{1-4 \nu}\left[\frac{113}{8}-\frac{2545}{56} \nu+\frac{918}{7} \nu^{2}-\left(\frac{699}{28}-\frac{495}{28} \nu\right) \nu\left(C_{Q A}+C_{Q B}\right)\right. \\
& \left.\left.-\frac{89}{7} \nu X_{A B}\left(C_{Q A}-C_{Q B}\right)\right] \hat{S} \hat{S}_{*}\right\} u^{7 / 2}+\mathcal{O}\left[u^{4}\right] \text {. }
\end{aligned}
$$




$$
\begin{aligned}
u(j)= & \frac{1}{j^{2}}+\frac{3}{j^{4}}-6\left(\hat{S}+\frac{3}{4} \hat{S}_{*}\right) \frac{1}{j^{5}} \\
& +\left\{18-3 \nu+\frac{1}{1-4 \nu}\left[-4 \nu+(1-2 \nu)\left(C_{Q A}+C_{Q B}\right)+X_{A B}\left(C_{Q A}-C_{Q B}\right)\right] \hat{S}^{2}\right. \\
& +\frac{1}{1-4 \nu}\left[-4 \nu+(1-2 \nu)\left(C_{Q A}+C_{Q B}\right)-X_{A B}\left(C_{Q A}-C_{Q B}\right)\right] \hat{S}_{*}^{2}+ \\
& \left.+\frac{4}{1-4 \nu}\left[1-2 \nu-\nu\left(C_{Q A}+C_{Q B}\right)\right] \hat{S} \hat{S}_{*}\right\} \frac{1}{j^{6}}+\left[\left(-69+\frac{5}{2} \nu\right) \hat{S}+\left(-\frac{189}{4}+3 \nu\right) \hat{S}_{*}\right] \frac{1}{j^{7}} \\
& +\left\{135+\left(-\frac{311}{3}+\frac{41}{16} \pi^{2}\right) \nu\right. \\
& +\frac{1}{1-4 \nu}\left[62-\frac{619}{2} \nu+\left(16-\frac{69}{2} \nu\right)\left(C_{Q A}+C_{Q B}\right)+\left(16-\frac{5}{2} \nu\right) X_{A B}\left(C_{Q A}-C_{Q B}\right)\right] \hat{S}^{2} \\
& +\frac{1}{1-4 \nu}\left[\frac{117}{4}-\frac{375}{2} \nu+\left(\frac{27}{2}-\frac{49}{2} \nu\right)\left(C_{Q A}+C_{Q B}\right)-\left(\frac{27}{2}+\frac{5}{2} \nu\right) X_{A B}\left(C_{Q A}-C_{Q B}\right)\right] \hat{S}_{*}^{2} \\
& \left.+\frac{1}{1-4 \nu}\left[147-465 \nu+-59 \nu\left(C_{Q A}+C_{Q B}\right)-5 \nu X_{A B}\left(C_{Q A}-C_{Q B}\right)\right] \hat{S} \hat{S}_{*}\right\} \frac{1}{j^{8}} \\
& +\left[\left(-\frac{3069}{4}+172 \nu+\frac{5}{8} \nu^{2}\right) \hat{S}+\left(-\frac{2007}{4}+\frac{585}{4} \nu+\frac{15}{16} \nu^{2}\right) \hat{S}_{*}\right] \frac{1}{j^{9}} \\
& +\left\{\frac { 1 } { 1 - 4 \nu } \left[\frac{5119}{2}-\frac{503105}{56} \nu+\frac{12555}{14} \nu^{2}-\left(\frac{10149}{14}-\frac{1251}{14} \nu\right) \nu\left(C_{Q A}+C_{Q B}\right)\right.\right. \\
& \left.\left.+\frac{1301}{14} \nu X_{A B}\left(C_{Q A}-C_{Q B}\right)\right] \hat{S} \hat{S}_{*}\right\} \frac{1}{j^{10}}+\mathcal{O}\left[j^{-11}\right] . \\
& +\frac{1}{1-4 \nu}\left[\frac{19223}{14}-\frac{88953}{14} \nu+\frac{6855}{14} \nu^{2}+\left(\frac{5725}{28}-\frac{26753}{56} \nu+\frac{1251}{28} \nu^{2}\right)\left(C_{Q A}+C_{Q B}\right)\right. \\
& \left.+\left(\frac{5725}{28}-\frac{3853}{56} \nu\right) X_{A B}\left(C_{Q A}-C_{Q B}\right)\right] \hat{S}^{2}+\frac{1}{1-4 \nu}\left[\frac{4653}{8}-\frac{12659}{4} \nu+\frac{5553}{14} \nu^{2}\right. \\
& \left.\left.+158-\frac{2335}{8} \nu+\frac{1251}{28} \nu^{2}\right)\left(C_{Q A}+C_{Q B}\right)-\left(158+\frac{193}{8} \nu\right) X_{A B}\left(C_{Q A}-C_{Q B}\right)\right] \hat{S}_{*}^{2} \\
& \left.+64 \gamma-\frac{31921}{1024} \pi^{2}+128 \log (2)+64 \log (1 / j)\right) \nu+\left(\frac{1321}{12}-\frac{205}{64} \pi^{2} \nu^{2}\right.
\end{aligned}
$$

Finally, the gauge-invariant link between the binding energy and the orbital angular momentum becomes 


$$
\begin{aligned}
& E_{b}(j)=-\frac{1}{2 j^{2}}\left\{1+\frac{1}{4}(9+\nu) \frac{1}{j^{2}}-4\left(\hat{S}+\frac{3}{4} \hat{S}_{*}\right) \frac{1}{j^{3}}\right. \\
& +\left\{\frac{1}{8}\left(81-7 \nu+\nu^{2}\right)+\frac{1}{1-4 \nu}\left[-2 \nu+\left(\frac{1}{2}-\nu\right)\left(C_{Q A}+C_{Q B}\right)+\frac{1}{2} X_{A B}\left(C_{Q A}-C_{Q B}\right)\right] \hat{S}^{2}\right. \\
& +\frac{1}{1-4 \nu}\left[-2 \nu+\left(\frac{1}{2}-\nu\right)\left(C_{Q A}+C_{Q B}\right)-\frac{1}{2} X_{A B}\left(C_{Q A}-C_{Q B}\right)\right] \hat{S}_{*}^{2}+ \\
& \left.+\frac{2}{1-4 \nu}\left[1-2 \nu-\nu\left(C_{Q A}+C_{Q B}\right)\right] \hat{S} \hat{S}_{*}\right\} \frac{1}{j^{4}}+\left[\left(-36-\frac{3}{4} \nu\right) \hat{S}+-\frac{99}{4} \hat{S}_{*}\right] \frac{1}{j^{5}} \\
& +\left\{\frac{3861}{64}-\left(\frac{8833}{192}-\frac{41}{32} \pi^{2}\right) \nu-\frac{5}{32} \nu^{2}+\frac{5}{64} \nu^{3}\right. \\
& +\frac{1}{1-4 \nu}\left[32-152 \nu-\nu^{2}+\left(\frac{25}{4}-\frac{53}{4} \nu-\frac{\nu^{2}}{2}\right)\left(C_{Q A}+C_{Q B}\right)+\left(\frac{25}{4}-\frac{3}{4} \nu\right) X_{A B}\left(C_{Q A}-C_{Q B}\right)\right] \hat{S}^{2} \\
& +\frac{1}{1-4 \nu}\left[\frac{63}{4}-87 \nu-\nu^{2}+\left(\frac{21}{4}-\frac{37}{4} \nu-\frac{\nu^{2}}{2}\right)\left(C_{Q A}+C_{Q B}\right)-\left(\frac{21}{4}+\frac{5}{4} \nu\right) X_{A B}\left(C_{Q A}-C_{Q B}\right)\right] \hat{S}_{*}^{2} \\
& \left.+\frac{1}{1-4 \nu}\left[69-227 \nu-2 \nu^{2}-(23+\nu) \nu\left(C_{Q A}+C_{Q B}\right)-2 \nu X_{A B}\left(C_{Q A}-C_{Q B}\right)\right] \hat{S} \hat{S}_{*}\right\} \frac{1}{j^{6}} \\
& +\left[\left(-\frac{1701}{8}+\frac{3597}{4} \nu-195 \nu^{2}\right) \hat{S}+\left(-\frac{4295}{16}+\frac{411}{8} \nu-\frac{5}{16} \nu^{2}\right) \hat{S}_{*}\right] \frac{1}{j^{7}} \\
& +\left\{\frac{53703}{128}-\left(\frac{989911}{1920}+\frac{128}{5} \gamma-\frac{6581}{512} \pi^{2}+\frac{256}{5} \log (2)+\frac{128}{5} \log (1 / j)\right) \nu+\left(\frac{8875}{384}-\frac{41}{64} \pi^{2}\right) \nu^{2}\right. \\
& -\frac{3}{64} \nu^{3}+\frac{7}{128} \nu^{4}+\frac{1}{1-4 \nu}\left[\frac{8013}{14}-\frac{71545}{28} \nu+\frac{2575}{28} \nu^{2}-\frac{3}{4} \nu^{3}+\left(\frac{7449}{112}-\frac{17119}{112} \nu+\frac{975}{112} \nu^{2}-\frac{3}{8} \nu^{3}\right)\left(C_{Q A}+C_{Q B}\right)\right. \\
& \left.+\left(\frac{7449}{112}-\frac{2221}{112} \nu-\frac{5}{16} \nu^{2}\right) X_{A B}\left(C_{Q A}-C_{Q B}\right)\right] \hat{S}^{2}+\frac{1}{1-4 \nu}\left[252-\frac{10203}{8} \nu+\frac{2757}{28} \nu^{2}-\frac{3}{4} \nu^{3}\right. \\
& \left.+\left(\frac{819}{16}-\frac{1473}{16} \nu+\frac{1199}{112} \nu^{2}-\frac{3}{8} \nu^{3}\right)\left(C_{Q A}+C_{Q B}\right)-\left(\frac{819}{16}+\frac{165}{16} \nu+\frac{11}{16} \nu^{2}\right) X_{A B}\left(C_{Q A}-C_{Q B}\right)\right] \hat{S}_{*}^{2} \\
& +\frac{1}{1-4 \nu}\left[\frac{4041}{4}-\frac{201927}{56} \nu+\frac{5661}{28} \nu^{2}-\frac{3}{2} \nu^{3}-\left(\frac{6591}{28}-\frac{533}{28} \nu+\frac{3}{4} \nu^{2}\right) \nu\left(C_{Q A}+C_{Q B}\right)\right. \\
& \left.\left.\left.-\left(\frac{429}{14}+\nu\right) \nu X_{A B}\left(C_{Q A}-C_{Q B}\right)\right] \hat{S} \hat{S}_{*}\right\} \frac{1}{j^{8}}+\mathcal{O}\left[j^{-9}\right]\right\} \text {. }
\end{aligned}
$$

[1] B. P. Abbott et al. (Virgo, LIGO Scientific), Phys. Rev. Lett. 119, $16 \overline{1101}$ (2017), arXiv:1710.05832 [gr-qc].

[2] B. P. Abbott et al. (Virgo, LIGO Scientific), (2018),

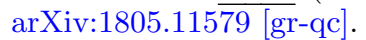

[3] B. P. Abbott et al. (Virgo, LIGO Scientific), (2018), arXiv:1805.11581 [gr-qc].

[4] T. Damour, in Gravitational Radiation, edited by N. Deruelle and T. Piran (North-Holland, Amsterdam, 1983) pp. 59-144.

[5] E. E. Flanagan and T. Hinderer, Phys.Rev. D77, 021502 (2008), arXiv:0709.1915 [astro-ph].

[6] T. Damour and A. Nagar, Phys. Rev. D80, 084035 (2009), arXiv:0906.0096 [gr-qc].
[7] T. Damour, A. Nagar, and L. Villain, Phys.Rev. D85, 123007 (2012), arXiv:1203.4352 [gr-qc].

[8] E. Poisson, Phys. Rev. D57, 5287 (1998), arXiv:grqc/9709032 [gr-qc].

[9] I. Harry and T. Hinderer, Class. Quant. Grav. 35, 145010 (2018), arXiv:1801.09972 [gr-qc].

[10] N. V. Krishnendu, K. G. Arun, and C. K. Mishra, Phys. Rev. Lett. 119, 091101 (2017), arXiv:1701.06318 [gr-qc].

[11] N. V. Krishnendu, C. K. Mishra, and K. G. Arun, (2018), arXiv:1811.00317 [gr-qc].

[12] J. Steinhoff, T. Hinderer, A. Buonanno, and A. Taracchini, Phys. Rev. D94, 104028 (2016), arXiv:1608.01907 [gr-qc]. 
[13] A. Nagar et al., (2018), arXiv:1806.01772 [gr-qc].

[14] T. Dietrich, S. Bernuzzi, and W. Tichy, Phys. Rev. D96, 121501 (2017), arXiv:1706.02969 [gr-qc].

[15] T. Dietrich et al., (2018), arXiv:1804.02235 [gr-qc].

[16] F. Messina and A. Nagar, Phys. Rev. D95, 124001 (2017), [Erratum: Phys. Rev.D96,no.4,049907(2017)], arXiv:1703.08107 [gr-qc].

[17] T. Marchand, L. Blanchet, and G. Faye, Class. Quant. Grav. 33, 244003 (2016), arXiv:1607.07601 [gr-qc].

[18] S. Bernuzzi, M. Thierfelder, and B. Brügmann, Phys.Rev. D85, 104030 (2012), arXiv:1109.3611 [gr-qc].

[19] S. Bernuzzi, A. Nagar, M. Thierfelder, and B. Brügmann, Phys.Rev. D86, 044030 (2012), arXiv:1205.3403 [gr-qc].

[20] D. Radice, L. Rezzolla, and F. Galeazzi, Mon.Not.Roy.Astron.Soc. 437, L46 (2014), arXiv:1306.6052 [gr-qc].

[21] S. Bernuzzi, A. Nagar, T. Dietrich, and T. Damour, Phys.Rev.Lett. 114, 161103 (2015), arXiv:1412.4553 [grqc].

[22] D. Radice, L. Rezzolla, and F. Galeazzi, Proceedings, Numerical Modeling of Space Plasma Flows ASP Conf. Ser. 498, 121 (2015), arXiv:1502.00551 [grqc].

[23] S. Bernuzzi and T. Dietrich, Phys. Rev. D94, 064062 (2016), arXiv:1604.07999 [gr-qc].

[24] D. Radice, S. Bernuzzi, and C. D. Ott, Phys. Rev. D94, 064011 (2016), arXiv:1603.05726 [gr-qc].

[25] S. Bernuzzi, T. Dietrich, W. Tichy, and B. Brügmann, Phys.Rev. D89, 104021 (2014), arXiv:1311.4443 [gr-qc].

[26] T. Dietrich, S. Bernuzzi, M. Ujevic, and W. Tichy, Phys. Rev. D95, 044045 (2017), arXiv:1611.07367 [gr-qc].

[27] M. Levi and J. Steinhoff, JCAP 1601, 008 (2016), arXiv:1506.05794 [gr-qc].

[28] M. Levi and J. Steinhoff, JCAP 1601, 011 (2016), arXiv:1506.05056 [gr-qc].

[29] M. Levi and J. Steinhoff, (2016), arXiv:1607.04252 [grqc].

[30] G. Schaefer and P. Jaranowski, Living Rev. Rel. 21, 7 (2018), arXiv:1805.07240 [gr-qc].

[31] T. Damour and A. Nagar, Phys.Rev. D90, 044018 (2014), arXiv:1406.6913 [gr-qc].

[32] A. Nagar and P. Rettegno, (2018), arXiv:1805.03891 [grqc].

[33] A. Nagar and A. Shah, Phys. Rev. D94, 104017 (2016), arXiv:1606.00207 [gr-qc].

[34] F. Messina, A. Maldarella, and A. Nagar, Phys. Rev. D97, 084016 (2018), arXiv:1801.02366 [gr-qc].

[35] T. Damour and A. Nagar, Phys.Rev. D90, 024054 (2014), arXiv:1406.0401 [gr-qc].

[36] J. Hartung and J. Steinhoff, Phys. Rev. D83, 044008 (2011), arXiv:1011.1179 [gr-qc].

[37] S. Balmelli and P. Jetzer, Phys. Rev. D91, 064011 (2015), arXiv:1502.01343 [gr-qc].

[38] T. Damour, P. Jaranowski, and G. Schäfer, Phys.Rev. D78, 024009 (2008), arXiv:0803.0915 [gr-qc].

[39] A. Nagar, Phys.Rev. D84, 084028 (2011), arXiv:1106.4349 [gr-qc].

[40] A. Nagar, T. Damour, C. Reisswig, and D. Pollney, Phys. Rev. D93, 044046 (2016), arXiv:1506.08457 [gr-qc].

[41] S. Marsat, Class. Quant. Grav. 32, 085008 (2015), arXiv:1411.4118 [gr-qc].

[42] S. Balmelli and T. Damour, Phys. Rev. D92, 124022 (2015), arXiv:1509.08135 [gr-qc].
[43] W. G. Laarakkers and E. Poisson, Astrophys. J. 512, 282 (1998), arXiv:gr-qc/9709033.

[44] G. Pappas and T. A. Apostolatos, Phys. Rev. Lett. 108, 231104 (2012), arXiv:1201.6067 [gr-qc].

[45] G. Pappas and T. A. Apostolatos, (2012), arXiv:1211.6299 [gr-qc].

[46] K. Yagi, K. Kyutoku, G. Pappas, N. Yunes, and T. A. Apostolatos, Phys. Rev. D89, 124013 (2014), arXiv:1403.6243 [gr-qc].

[47] K. Yagi and N. Yunes, Science 341, 365 (2013), arXiv:1302.4499 [gr-qc].

[48] K. Yagi and N. Yunes, Phys.Rev. D88, 023009 (2013), arXiv:1303.1528 [gr-qc].

[49] T. Damour, M. Soffel, and C.-m. Xu, Phys. Rev. D47, 3124 (1993).

[50] T. Hinderer, Astrophys.J. 677, 1216 (2008), arXiv:0711.2420 [astro-ph].

[51] T. Binnington and E. Poisson, Phys. Rev. D80, 084018 (2009), arXiv:0906.1366 [gr-qc].

[52] E. Barausse and A. Buonanno, Phys.Rev. D81, 084024 (2010), arXiv:0912.3517 [gr-qc].

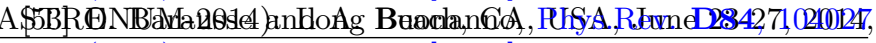
(2011), arXiv:1107.2904 [gr-qc].

[54] A. Taracchini, Y. Pan, A. Buonanno, E. Barausse, M. Boyle, et al., Phys.Rev. D86, 024011 (2012), arXiv:1202.0790 [gr-qc].

[55] T. Hinderer, (private communication, 2018).

[56] S. Marsat and A. Bohé, (private communication, 2018).

[57] T. Damour, B. R. Iyer, and B. S. Sathyaprakash, Phys. Rev. D63, 044023 (2001), [Erratum: Phys. Rev.D72,029902(2005)], arXiv:gr-qc/0010009 [gr-qc].

[58] T. Damour, B. R. Iyer, and A. Nagar, Phys. Rev. D79, 064004 (2009), arXiv:0811.2069 [gr-qc].

[59] L. Bernard, L. Blanchet, A. Bohé, G. Faye, and S. Marsat, Phys. Rev. D95, 044026 (2017), arXiv:1610.07934 [gr-qc].

[60] T. Damour, P. Jaranowski, and G. Schäfer, Phys. Rev. D93, 084014 (2016), arXiv:1601.01283 [gr-qc].

[61] T. Damour, P. Jaranowski, and G. Schfer, Phys. Rev. D91, 084024 (2015), arXiv:1502.07245 [gr-qc].

[62] T. Damour, P. Jaranowski, and G. Schfer, Phys. Rev. D89, 064058 (2014), arXiv:1401.4548 [gr-qc].

[63] L. Blanchet, Living Rev. Relativity 17, 2 (2014), arXiv:1310.1528 [gr-qc].

[64] A. Bohé, G. Faye, S. Marsat, and E. K. Porter, Class. Quant. Grav. 32, 195010 (2015), arXiv:1501.01529 [grqc].

[65] R. Cotesta, A. Buonanno, A. Bohé, A. Taracchini, I. Hinder, and S. Ossokine, (2018), arXiv:1803.10701 [gr-qc].

[66] G. Faye, L. Blanchet, and B. R. Iyer, Class. Quant. Grav. 32, 045016 (2015), arXiv:1409.3546 [gr-qc].

[67] A. Bohé, S. Marsat, and L. Blanchet, Class. Quant. Grav. 30, 135009 (2013), arXiv:1303.7412 [gr-qc].

[68] S. Marsat, A. Bohé, L. Blanchet, and A. Buonanno, Class. Quant. Grav. 31, 025023 (2014), arXiv:1307.6793 [gr-qc].

[69] R. Fujita, PTEP 2015, 033E01 (2015), arXiv:1412.5689 [gr-qc].

[70] S. Akcay, S. Bernuzzi, F. Messina, A. Nagar, N. Ortiz, and P. Rettegno, (2018), arXiv:1812.02744 [gr-qc].

[71] A. Buonanno and T. Damour, Phys. Rev. D59, 084006 (1999), arXiv:gr-qc/9811091.

[72] A. Buonanno and T. Damour, Phys. Rev. D62, 064015 (2000), arXiv:gr-qc/0001013. 
[73] T. Damour and A. Nagar, Phys. Rev. D77, 024043 (2008), arXiv:0711.2628 [gr-qc].

[74] T. Damour, A. Nagar, and S. Bernuzzi, Phys.Rev. D87, 084035 (2013), arXiv:1212.4357 [gr-qc].
[75] B. D. Lackey, S. Bernuzzi, C. R. Galley, J. Meidam, and C. Van Den Broeck, Phys. Rev. D95, 104036 (2017), arXiv:1610.04742 [gr-qc]. 